\title{
Universal Compressed Sensing
}

\author{
Shirin Jalali, H. Vincent Poor
}

\begin{abstract}
The main promise of compressed sensing is accurate recovery of high-dimensional structured signals from an underdetermined set of randomized linear projections. Several types of structure such as sparsity and low-rankness have already been explored in the literature. For each type of structure, a recovery algorithm has been developed based on the properties of signals with that type of structure. Such algorithms recover any signal complying with the assumed model from its sufficient number of linear projections. However, in many practical situations the underlying structure of the signal is not known, or is only partially known. Moreover, it is desirable to have recovery algorithms that can be applied to signals with different types of structure.

In this paper, the problem of developing universal algorithms for compressed sensing of stochastic processes is studied. First, Rényi's notion of information dimension (ID) is generalized to analog stationary processes. This provides a measure of complexity for such processes and is connected to the number of measurements required for their accurate recovery. Then a minimum entropy pursuit (MEP) optimization approach is proposed, and it is proven that it can reliably recover any stationary process satisfying some mixing constraints from sufficient number of randomized linear measurements, without having any prior information about the distribution of the process. It is proved that a Lagrangian-type approximation of the MEP optimization problem, referred to as Lagrangian-MEP problem, is identical to a heuristic implementable algorithm proposed by Baron et al. It is shown that for the right choice of parameters the Lagrangian-MEP algorithm, in addition to having the same asymptotic performance as MEP optimization, is also robust to the measurement noise. For memoryless sources with a discrete-continuous mixture distribution, the fundamental limits of the minimum number of required measurements by a non-universal compressed sensing decoder is characterized by Wu et al. For such sources, it is proved that there is no loss in universal coding, and both the MEP and the Lagrangian-MEP asymptotically achieve the optimal performance.
\end{abstract}

\section{INTRODUCTION}

Consider the fundamental problem of compressed sensing (CS): a signal $x_{o}^{n} \in \mathbb{R}^{n}$ is measured through a data acquisition process modeled by a linear projection system: $y_{o}^{m}=A x_{o}^{n}$, where $A \in \mathbb{R}^{m \times n}$ denotes the measurement matrix. The signal $x_{o}^{n}$ is usually high-dimensional, and the number of measurements is much smaller than the ambient dimension of the signal, i.e., $m \ll n$. The decoder is interested in recovering $x_{o}^{n}$ from the measurements $y_{o}^{m}$. Since the system of linear equations described by $y_{o}^{m}=A x^{n}$ has infinitely many solutions, without any side information, clearly it is impossible to recover $x_{o}^{n}$ from $y_{o}^{m}$. However, with some extra information about the structure of $x_{o}^{n}$, one might be able to recover $x_{o}^{n}$ from $y_{o}^{m}$ reliably. Intuitively, having this extra information enables the decoder to, among the signals that satisfy the measurement constraints, search for the one that is (more) consistent with the structure model. For instance for sparse signals, i.e., signal with $k=\left\|x_{o}^{n}\right\|_{0} \ll n,{ }^{1}$ the decoder

${ }^{1}$ For $x^{n} \in \mathbb{R}^{n},\left\|x_{o}^{n}\right\|_{0} \triangleq\left|\left\{i: x_{i} \neq 0\right\}\right|$. 
might try to find the signal with minimum $\ell_{0}$-norm among the signals that satisfy $A x^{n}=y_{o}^{m}$. In that case the reconstruction signal will be

$$
\hat{x}_{o}^{n}=\underset{A x^{n}=y_{o}^{n}}{\arg \min }\left\|x^{n}\right\|_{0}
$$

While this optimization is not practical, it has well-known approximations that can be implemented efficiently [1]-[3]. This result can also be extended to several other structures such as group-sparsity and low-rankness. (Refer to [4]-[11] for some examples of the other structures studied in the literature.) In each of these cases, the signal is known to have some specific structure, and the decoder exploits this side information to recover the signal from its under-sampled set of linear projections.

Structures that are already studied in the compressed sensing literature are often simple models such as sparsity. However, natural signals typically exhibit much more complicated and diverse patterns. Therefore, it is desirable to have a recovery algorithm that can be applied to sources with diverse structures without having some prior information about the source model. Such algorithms are referred to as universal algorithms in the information theory literature. More formally universal algorithms are defined as algorithms that achieve the optimal performance without knowing the source distribution. Existence of such algorithms has been proved for several different problems such as compression [12]-[16], denoising [17], [18] and prediction [19], [20].

In order to develop a universal compressed sensing algorithm, there are some fundamental questions that need to be addressed: What does it mean for an analog ${ }^{2}$ signal to be of low complexity or structured? How can the structure or the complexity of an analog signal be measured? Is it possible to design a universal compressed sensing decoder that is able to recover structured signals from their randomized linear projections ${ }^{3}$ without knowing the underlying structure of the signal?

The problem of universal compressed sensing has already been studied in the literature [21]-[25]. In [21] and [22], the authors propose a heuristic implementable algorithm for universal compressed sensing of stochastic processes. In [23], the authors define the Kolmogorov information dimension (KID) of a deterministic analog signal as a measure of its complexity. The KID of a signal $x_{o}^{n}$ is defined as the growth rate of the Kolmogorov complexity of the quantized version of $x_{o}^{n}$ normalized by the log of the number of quantization levels, as the number of quantization levels grows to infinity. Employing this measure of complexity, the authors in [23] and [25] propose a minimum complexity pursuit (MCP) optimistion as a universal signal recovery decoder. MCP is based on Occam's razor [26], i.e., among all signals satisfying the linear measurement constraints, MCP seeks the one with the lowest complexity. While MCP proves the existence of universal compressed sensing algorithms, it is not an implementable algorithm, since it is based on minimizing Kolmogorov complexity [27], [28], which is not computable.

In this paper we focus on stochastic signals and develop an implementable algorithm for universal compressed sensing of stochastic processes. To achieve this goal, we first need to develop a measure of complexity for stochastic

\footnotetext{
${ }^{2}$ Throughout the paper, an analog signal refers to a continuous-alphabet discrete-time signal.

${ }^{3}$ Throughout the paper, linear measurements acquired by a measurement matrix generated from a random distribution is denoted by randomized linear projections or randomized linear measurements.
} 
processes that differentiates between different processes in terms of their complexities. To define such a measure, we extend the Rényi's notion of the information dimension of an analog random variable [29] to define the information dimension of a stochastic process. As we will show, this extension is consistent with Rényi's information dimension such that the information dimension of a memoryless stationary process $X=\left\{X_{i}\right\}_{i=1}^{\infty}$ is equal to the Rényi information dimension of its first-order marginal distribution $\left(X_{1}\right)$. It has recently been proved that for independent and identically distributed (i.i.d.) processes with a mixture of discrete-continuous distribution, their Rényi information dimension characterizes the fundamental limits of (non-universal) compressed sensing [30].

Again consider the basic problem of compressed sensing: $X_{o}^{n}$ is generated by an analog stationary process $X=\left\{X_{i}\right\}_{i=1}^{\infty}$, the decoder observes its linear projections $Y_{o}^{m}=A X_{o}^{n}$, where $m<n$, and is interested in recovering $X_{o}^{n}$. To recover $X_{o}^{n}$ from $Y_{o}^{m}$, in the same spirit of the MCP algorithm, we propose minimum entropy pursuit (MEP) optimization, which among all the signals $x^{n}$ satisfying the measurement constraint $Y_{o}^{n}=A x^{n}$, outputs the one whose quantized version has the minimum conditional empirical entropy. We prove that, asymptotically, for a proper choice of the quantization level and the order of the conditional empirical entropy, and having slightly more than the (upper) information dimension of the process times the ambient dimension of the process randomized linear measurements, MEP presents an asymptotically lossless estimate of $X_{o}^{n}$. While MEP is not easy to implement, we also present an implementable version with the same asymptotic performance guarantees as MEP. The implementable approximation of the MEP optimization, which we refer to as Lagrangian-MEP, is identical to the heuristic algorithm proposed and implemented in [21] and [22] for universal compressed sensing. We prove that for the right choice of parameters, the Lagrangian-MEP algorithm has the same asymptotic performance as MEP and in addition is also robust to measurement noise. That is, the asymptotic performance of the Lagrangian-MEP algorithm does not change when the measurement vector is corrupted by a small-enough measurement noise vector. For memoryless sources with a discrete-continuous mixture distribution, we show that there is no loss in the performance due to universal coding, and both the MEP optimization and the Lagrangian-MEP algorithm achieve the optimal performance derived in $[30]$.

The organization of the paper is as follows. Section II introduces the notation used in the paper and reviews some related background. Section III presents an overview of the main contributions of the paper. In Section IV, we first generalize the Rényi's notion of the information dimension of a random variable [29] and define the information dimension of a stationary process. In Section V, we introduce the MEP optimization for universal compressed sensing, and also provide an implementable version of MEP, namely Lagrangian-MEP, which is the same heuristic algorithm proposed in [21] and [22], and prove its optimality and its robustness to measurement noise. The proofs of all of the main results are given in Section VI. Section VII concludes the paper.

\section{BACKGROUND}

In this section we first introduce the notation that is used throughout the paper. Then, we review two basic concepts, namely empirical distribution and universal lossless compression, which we employ in developing our results on universal compressed sensing. 


\section{A. Notation}

Calligraphic letters such as $\mathcal{X}$ and $\mathcal{Y}$ denote sets. For a finite set $\mathcal{X}$, let $|\mathcal{X}|$ denote the size of $\mathcal{X}$. Given vectors $u^{n}, v^{n} \in \mathbb{R}^{n}$, let $\left\langle u^{n}, v^{n}\right\rangle$ denote their inner product, i.e., $\left\langle u^{n}, v^{n}\right\rangle \triangleq \sum_{i=1}^{n} u_{i} v_{i}$. Also, $\left\|u^{n}\right\|_{2} \triangleq\left(\sum_{i=1}^{n} u_{i}^{2}\right)^{0.5}$ denotes the $\ell_{2}$-norm of $u^{n}$. For $1 \leq i \leq j \leq n, u_{i}^{j} \triangleq\left(u_{i}, u_{i+1}, \ldots, u_{j}\right)$. To simplify the notation, $u^{j} \triangleq u_{1}^{j}$. The set of all finite-length binary sequences is denoted by $\{0,1\}^{*}$, i.e., $\{0,1\}^{*} \triangleq \cup_{n \geq 1}\{0,1\}^{n}$. Similarly, $\{0,1\}^{\infty}$ denotes the set of infinite-length binary sequences. Throughout the paper log refers to logarithm to the basis of 2 and $\ln$ refers to the natural logarithm.

Random variables are represented by upper-case letters such as $X$ and $Y$. The alphabet of the random variable $X$ is denoted by $\mathcal{X}$. Given a sample space $\Omega$ and event $\mathcal{A} \subseteq \Omega, \mathbb{1}_{\mathcal{A}}$ denotes the indicator function of $\mathcal{A}$. Given $x \in \mathbb{R}, \delta_{x}$ denotes the Dirac measure with an atom at $x$.

Given a real number $x \in \mathbb{R},\lfloor x\rfloor(\lceil x\rceil)$ denotes the largest (the smallest) integer number smaller (larger) than $x$. Further, $[x]_{b}$ denotes the $b$-bit quantized version of $x$ that results from taking the first $b$ bits in the binary expansion of $x$. That is, for $x=\lfloor x\rfloor+\sum_{i=1}^{\infty} 2^{-i}(x)_{i}$, where $(x)_{i} \in\{0,1\}$,

$$
[x]_{b} \triangleq\lfloor x\rfloor+\sum_{i=1}^{b} 2^{-i}(x)_{i} .
$$

Also, for $x^{n} \in \mathbb{R}^{n}$, define

$$
\left[x^{n}\right]_{b} \triangleq\left(\left[x_{1}\right]_{b}, \ldots,\left[x_{n}\right]_{b}\right)
$$

For a positive integer $\ell$, let

$$
\langle x\rangle_{\ell} \triangleq \frac{\lfloor\ell x\rfloor}{\ell} .
$$

By this definition, $\langle x\rangle_{\ell}$ is a finite-alphabet approximation of the random variable $X$, such that $0<x-\langle x\rangle_{\ell} \leq \frac{1}{\ell}$.

\section{B. Conditional empirical entropy}

Consider a stochastic process $X=\left\{X_{i}\right\}_{i=1}^{\infty}$, with finite alphabet $\mathcal{X}$ and probability measure $\mu(\cdot)$. The entropy rate of a stationary process $X$ is defined as

$$
\bar{H}(X) \triangleq \lim _{n \rightarrow \infty} \frac{H\left(X_{1}, \ldots, X_{n}\right)}{n} .
$$

The $k$-th order empirical distribution induced by $x^{n} \in \mathcal{X}^{n}, p_{k}\left(. \mid x^{n}\right)$ is defined as

$$
p_{k}\left(a^{k} \mid x^{n}\right)=\frac{\left|\left\{i: x_{i-k}^{i-1}=a^{k}, 1 \leq i \leq n\right\}\right|}{n},
$$

where we make a circular assumption such that $x_{j}=x_{j+n}$, for $j \leq 0$.

Definition 1. The conditional empirical entropy induced by $x^{n} \in \mathcal{X}^{n}, \hat{H}_{k}\left(x^{n}\right)$, is equal to $H\left(U_{k+1} \mid U^{k}\right)$, where $U^{k+1} \sim p_{k+1}\left(\cdot \mid x^{n}\right)$.

For a stationary finite-alphabet process $X$, if $k$ grows to infinity as $k=o(\log n), \hat{H}_{k}\left(X^{n}\right)$ converges, almost 
surely, to the entropy rate of the process $X$, i.e., $\hat{H}_{k}\left(X^{n}\right) \rightarrow \bar{H}(X)$, almost surely [31]. Therefore, if we fix the size of the source alphabet, $\hat{H}_{k}$ is a universal estimator of the source entropy rate, which in turn is a measure of the source complexity.

\section{Universal lossless compression}

One of the most well-studied universal coding problems is the problem of universal lossless compression. The entropy rate of a stationary process characterizes the minimum number of bits per symbol required for its lossless compression. Universal lossless compression algorithms, such as the Lempel-Ziv algorithm [32], asymptotically spend the same number of bits per symbol for compressing any stationary ergodic process, without knowing its distribution. To design a universal lossless compression algorithm, similar to universal compressed sensing, one needs to develop a universal measure of complexity. The fundamental difference between the two problems is that while compressed sensing is mainly concerned with continuous-alphabet sources, lossless compression is concerned with discrete-alphabet processes. In the following, we briefly review the mathematical definition of a universal lossless compression algorithm.

Consider the problem of universal lossless compression of discrete stationary ergodic sources described as follows. A family of source codes $\left\{\mathcal{C}_{n}\right\}_{n \geq 1}$ consists of a sequence of codes corresponding to different blocklengths. Each code $\mathcal{C}_{n}$ in this family is defined by an encoder function $f_{n}$ and a decoder function $g_{n}$ such that

$$
f_{n}: \mathcal{X}^{n} \rightarrow\{0,1\}^{*}
$$

and

$$
g_{n}:\{0,1\}^{*} \rightarrow \hat{\mathcal{X}}^{n}
$$

Here $\hat{\mathcal{X}}$ denotes the reconstruction alphabet which is also assumed to be discrete and in many cases is equal to $\mathcal{X}$. The encoder $f_{n}$ maps each source block $X^{n}$ to a binary sequence of finite length, and the decoder $g_{n}$ maps the coded bits back to the signal space as $\hat{X}^{n}=g_{n}\left(f_{n}\left(X^{n}\right)\right)$. Let $l_{n}\left(f_{n}\left(X^{n}\right)\right)=\left|f_{n}\left(X^{n}\right)\right|$ denote the length of the binary sequence assigned to the sequence $X^{n}$. We assume that the codes are lossless (non-singular), i.e., $f_{n}\left(x^{n}\right) \neq f_{n}\left(\tilde{x}^{n}\right)$, for all $x^{n} \neq \tilde{x}^{n}$. A family of lossless codes is called universal, if

$$
\frac{1}{n} \mathrm{E}\left[l_{n}\left(f_{n}\left(X^{n}\right)\right)\right] \rightarrow \bar{H}(X)
$$

and $\mathrm{P}\left(X^{n} \neq \hat{X}^{n}\right) \rightarrow 0$, as $n$ grows to infinity, for any discrete stationary process $X$. A family of lossless codes is called point-wise universal, if

$$
\frac{1}{n} l_{n}\left(f_{n}\left(X^{n}\right)\right) \rightarrow \bar{H}(X)
$$

almost surely, for any discrete stationary ergodic process $X$. The Lempel-Ziv algorithm [32] is both a universal and a point-wise universal lossless compression algorithm. For a discrete-alphabet sequence $u^{n}, \ell_{\mathrm{LZ}}\left(u^{n}\right)$ denotes the length of encoded version of $u^{n}$ by the Lempel-Ziv algorithm. 


\section{OVERVIEW OF RESULTS}

In recovering a structured signal $x_{o}^{n}$ from undersampled linear measurements $y_{o}^{m}=A x_{o}^{n}, m<n$, non-universal algorithms look for the signal that complies with both the measurements and the known structure. For several types of structure, it has been proved that with enough measurements, this procedure yields a reliable estimate of the input vector $x_{o}^{n}$. In the universal compressed sensing problem, the decoder does not have any information about the structure or the distribution of the source. In the deterministic settings, the authors in [25] proved that universal compressed sensing is possible and proposed the KID measure of complexity and the MCP universal recovery algorithm. However, as explained earlier, both the MCP optimization and the KID measure are based on the notion of Kolmogorov complexity and hence are not computable. Moreover, KID measures the complexity of an individual sequence, not a stochastic process. The focus of this paper is on stationary processes. Therefore, as the first step, in Section IV, we develop a measure of complexity, referred to as ID, for stationary processes. After that, in Section $\mathrm{V}$ we focus on developing a universal compressed sensing algorithm. In Section V-A, we propose the MEP optimization, as a universal compressed sensing method, which does not require any prior knowledge about the source model. Then, in Section V-B, we study the theoretical performance of the MEP. In order to develop a universal compressed sensing algorithm for stochastic processes, an estimator of their ID based on the process realizations is required. Therefore, in Section V-B1, we study special stationary processes such as $\Psi^{*}$ mixing processes for which we are able to design such an estimator. Then, for such processes, in Section V-B2, we prove that for the right set of parameters, the normalized number of measurements required by the MEP or by its implementable version, namely, the Lagrangian-MEP, is slightly more than the ID of the source process.

\section{ID OF STATIONARY PROCESSES}

Consider an analog random variable $X$ and integer $n \in \mathbb{N}$. Rényi defined the upper and lower information dimensions of a random variable $X$ in terms of the entropy of $\langle X\rangle_{n}$ as

$$
\bar{d}(X)=\limsup _{n} \frac{H\left(\langle X\rangle_{n}\right)}{\log n},
$$

and

$$
\underline{d}(X)=\liminf _{n} \frac{H\left(\langle X\rangle_{n}\right)}{\log n},
$$

respectively [29]. If $\bar{d}(X)=\underline{d}(X)$, then the information dimension of the random variable $X$ is defined as

$$
d(X)=\lim _{n \rightarrow \infty} \frac{H\left(\langle X\rangle_{n}\right)}{\log n}
$$

While the Rényi information dimension measure can be used in measuring the complexity of memoryless continuous-alphabet sources, it cannot directly be applied to analog stationary processes with memory. For instance, a piecewise-constant signal generated by a stationary first-order Markov process is expected to be of low complexity. However, the complexity of such processes cannot be evaluated using the Rényi information dimension or the entropy rate function. In the following, we develop a measure of complexity for stationary analog sources. To achieve this 
goal, we carefully combine the definitions of the entropy rate and the Rényi information dimension, to capture both the source memory and the fact that the source is continuous-alphabet.

Define the $b$-bit quantized version of a stochastic process $X=\left\{X_{i}\right\}_{i=1}^{\infty}$ as $[X]_{b}=\left\{\left[X_{i}\right]_{b}\right\}_{i=1}^{\infty}$. Consider a stationary process $X=\left\{X_{i}\right\}_{i=1}^{\infty}$; then since $[X]_{b}$ is derived from a stationary coding of $X$, it is also a stationary process. We define the $k$-th order upper information dimension of a process $X$ as

$$
\bar{d}_{k}(X)=\limsup _{b \rightarrow \infty} \frac{H\left(\left[X_{k+1}\right]_{b} \mid\left[X^{k}\right]_{b}\right)}{b} .
$$

Similarly, the $k$-th order lower information dimension of $X$ is defined as

$$
\underline{d}_{k}(X)=\liminf _{b \rightarrow \infty} \frac{H\left(\left[X_{k+1}\right]_{b} \mid\left[X^{k}\right]_{b}\right)}{b} .
$$

Lemma 1. Both $\bar{d}_{k}(X)$ and $\underline{d}_{k}(X)$ are non-increasing in $k$.

Proof: For a stationary process $[X]_{b}$, for any value of $k$,

$$
\begin{aligned}
\frac{H\left(\left[X_{k+2}\right]_{b} \mid\left[X^{k+1}\right]_{b}\right)}{b} & \leq \frac{H\left(\left[X_{k+2}\right]_{b} \mid\left[X_{2}^{k+1}\right]_{b}\right)}{b} \\
& =\frac{H\left(\left[X_{k+1}\right]_{b} \mid\left[X^{k}\right]_{b}\right)}{b} .
\end{aligned}
$$

Therefore, taking liminf and limsup of both sides as $b$ grows to infinity yields the desired result.

Definition 2 (Upper/lower information dimension). For a stationary process $X$, if $\lim _{k \rightarrow \infty} \bar{d}_{k}(X)$ exists, we define the upper information dimension of process $X$ as

$$
\bar{d}_{o}(X)=\lim _{k \rightarrow \infty} \bar{d}_{k}(X)
$$

Similarly, if $\lim _{k \rightarrow \infty} \underline{d}_{k}(X)$ exists, the lower information dimension of process $X$ is defined as

$$
\underline{d}_{o}(X)=\lim _{k \rightarrow \infty} \underline{d}_{k}(X) .
$$

If $\underline{d}_{o}(X)=\bar{d}_{o}(X), d_{o}(X) \triangleq \underline{d}_{o}(X)=\bar{d}_{o}(X)$ is defined as the information dimension of the process $X$.

Lemma 2. Consider a stationary process $X$, with $\mathcal{X}=[l, u]$, where $l<u$ and $l, u \in \mathbb{R}$. Then, $\bar{d}_{k}(X) \leq 1$ and $\underline{d}_{k}(X) \leq 1$, for all $k$.

Proof: Note that $H\left(\left[X_{k+1}\right]_{b} \mid\left[X^{k}\right]_{b}\right) \leq \log \left((u-l) 2^{b}\right)=\log (u-l)+b$, for all $b$ and all $k$, and therefore,

$$
\frac{1}{b} H\left(\left[X_{k+1}\right]_{b} \mid\left[X^{k}\right]_{b}\right) \leq 1+\frac{\log (u-l)}{b} .
$$

Taking limsup and liminf of both sides as $b$ grows to infinity yields $\bar{d}_{k}(X) \leq 1$ and $\underline{d}_{k}(X) \leq 1$, for all $k$.

For stationary bounded processes, from Lemmas 1 and $2, \bar{d}_{k}(X)$ and $\underline{d}_{k}(X)$ are monotonic bounded sequences. Therefore, $\lim _{k \rightarrow \infty} \bar{d}_{k}(X)$ and $\lim _{k \rightarrow \infty} \underline{d}_{k}(X)$ both exist, and the upper and lower information dimensions of such 
processes are well-defined.

The entropy rate of a discrete stationary process can be defined either as (1) or equivalently as $\bar{H}(X)=$ $\lim _{k \rightarrow \infty} H\left(X_{k} \mid X^{k-1}\right)$. In the same spirit, the following lemma presents an equivalent representation of the upper information dimension of a process.

Lemma 3. For a stationary process $X$, with upper information dimension $\bar{d}_{o}(X)$,

$$
\bar{d}_{o}(X)=\lim _{k \rightarrow \infty} \frac{1}{k}\left(\limsup _{b \rightarrow \infty} \frac{H\left(\left[X^{k}\right]_{b}\right)}{b}\right) .
$$

The following proposition proves that the information dimension of stationary memoryless processes is equal to the Rényi information dimension of their first order marginal distribution. This implies that our notion of information dimension for stochastic processes is consistent with the Rényi's notion of information dimension for random variables.

Proposition 1. For an i.i.d. process $X=\left\{X_{i}\right\}_{i=1}^{\infty}, \bar{d}_{o}(X)\left(\underline{d}_{o}(X)\right)$ is equal to $\bar{d}\left(X_{1}\right)\left(\underline{d}\left(X_{1}\right)\right)$, the Rényi upper (lower) information dimension of $X_{1}$.

Proof: Since the process is memoryless, for any quantization level $b, H\left(\left[X_{k+1}\right]_{b} \mid\left[X^{k}\right]_{b}\right)=H\left(\left[X_{k+1}\right]_{b}\right)$ and since it is stationary, $H\left(\left[X_{k+1}\right]_{b}\right)=H\left(\left[X_{1}\right]_{b}\right)$. Therefore,

$$
\bar{d}_{k}(X)=\bar{d}_{0}(X)=\underset{b}{\limsup } \frac{H\left(\left[X_{1}\right]_{b}\right)}{b} .
$$

As proved in Proposition 2 of [30],

$$
\bar{d}\left(X_{1}\right)=\limsup _{b} \frac{H\left(\left\langle X_{1}\right\rangle_{2^{b}}\right)}{b} .
$$

Since $\left\langle X_{1}\right\rangle_{2^{b}}=\left[X_{1}\right]_{b}$, this yields the desired result.

To clarify the notion of information dimension, in the following we present several examples of different stationary processes and evaluate their information dimensions.

The following theorem, which follows from Theorem 3 of [29] combined with Proposition 1, characterizes the information dimension of i.i.d processes, whose components are drawn from a mixture of continuous and discrete distribution.

Theorem 1 (Theorem 3 in [29]). Consider an i.i.d. process $\left\{X_{i}\right\}_{i=1}^{\infty}$, where each $X_{i}$ is distributed according to

$$
(1-p) f_{d}+p f_{c}
$$

where $f_{d}$ and $f_{c}$ represent a discrete measure and an absolutely continuous measure, respectively. Also, $p \in[0,1]$ denotes the probability that $X_{i}$ is drawn from the continuous distribution $f_{c}$. Assume that $H\left(\left\lfloor X_{1}\right\rfloor\right)<\infty$. Then,

$$
\bar{d}_{o}(X)=\underline{d}_{o}(X)=d_{o}(X)=p
$$


From Theorem 1, for an i.i.d. process with components drawn from an absolutely continuous distribution ${ }^{4}$ the information dimension is equal to one. As a reminder, from Lemma 2, for sources with bounded alphabet, $d_{o}(X) \leq$ 1. Therefore, from Theorem 1, memoryless sources with an absolutely continuous distribution have maximum complexity. As $p$, the weight of the continuous component, decreases from one, the information dimension of the source, or equivalently its complexity, decreases as well.

Processes with piecewise constant realizations are one of the standard models in image processing, and are studied in various problems such as denoising and compressed sensing. Such processes can be modeled as a first-order Markov process. Theorem 3 evaluates the information dimension of such processes, and shows that their complexity depends on the rate of their jumps. Before that, Theorem 2 connects the information dimension of a Markov process of order $l$ to its $l$-th order information dimension.

Theorem 2. Consider a stationary Markov process $X$ of order $\ell$. Then,

$$
\limsup _{b \rightarrow \infty} \frac{H\left(\left[X_{l+1}\right]_{b} \mid X^{l}\right)}{b} \leq \bar{d}_{o}(X) \leq \bar{d}_{\ell}(X),
$$

and

$$
\liminf _{b \rightarrow \infty} \frac{H\left(\left[X_{l+1}\right]_{b} \mid X^{l}\right)}{b} \leq \underline{d}_{o}(X) \leq \underline{d}_{\ell}(X) .
$$

Proof: The upper bounds on both cases follow from Lemma 1. To prove the lower bound, note that for $k>l$,

$$
\begin{aligned}
\left.H\left(\left[X_{k+1}\right]_{b} \mid\left[X^{k}\right]_{b}\right]\right) & \left.\geq H\left(\left[X_{k+1}\right]_{b} \mid\left[X^{k}\right]_{b}\right], X_{k-l+1}^{k}\right) \\
& \stackrel{(a)}{=} H\left(\left[X_{k+1}\right]_{b} \mid X_{k-l+1}^{k}\right) \\
& \stackrel{(b)}{=} H\left(\left[X_{l+1}\right]_{b} \mid X^{l}\right)
\end{aligned}
$$

where $(a)$ holds because $X$ is a Markov process of order $l$ and therefore $\left[X^{k}\right]_{b} \rightarrow X_{k-l+1}^{k} \rightarrow\left[X_{k+1}\right]_{b}$. Equality (b) follows from the stationarity of $X$. Taking limsup of the both sides of (2), it follows that

$$
\bar{d}_{k}(X)=\limsup _{b \rightarrow \infty} \frac{\left.H\left(\left[X_{k+1}\right]_{b} \mid\left[X^{k}\right]_{b}\right]\right)}{b} \geq \limsup _{b \rightarrow \infty} \frac{H\left(\left[X_{l+1}\right]_{b} \mid X^{l}\right)}{b} .
$$

Similarly, taking liminf of the both sides yields the lower bound on $\underline{d}_{O}(X)$.

Theorem 3. Consider a first-order stationary Markov process $X=\left\{X_{i}\right\}_{i=1}^{\infty}$, such that conditioned on $X_{t-1}=$ $x_{t-1}, X_{t}$ has a mixture of discrete and absolutely continuous distribution equal to $(1-p) \delta_{x_{t-1}}+p f_{c}$, where $f_{c}$ represents the pdf of an absolutely continuous distribution over $[0,1]$ with bounded differential entropy. Then,

$$
d_{o}(X)=p
$$

As another example, Theorem 4 below considers a special type of auto-regressive Markov processes of order $l$, $l \in \mathbb{N}$, and evaluates their information dimension.

\footnotetext{
${ }^{4} \mathrm{~A}$ probability distribution is called absolutely continuous, if it has a probability density function (pdf).
} 
Theorem 4. Consider a stationary Markov process of order $l$ such that conditioned on $X_{t-l}^{t-1}=x_{t-l}^{t-1}, X_{t}$ is distributed as $\sum_{i=1}^{l} a_{i} x_{t-i}+Z_{t}$, where $a_{i} \in(0,1)$, for $i=1, \ldots, l$, and $Z_{t}$ is an i.i.d. process distributed according to $(1-p) \delta_{0}+p f_{c}$, where $f_{c}$ is the pdf of an absolutely continuous distribution. Let $\mathcal{Z}$ denote the support of $f_{c}$ and assume that there exists $0<\alpha<\beta<\infty$, such that $\alpha<f_{c}(z)<\beta$, for $z \in \mathcal{Z}$. Then,

$$
d_{o}(X)=p
$$

Finally, the last result of this section is concerned with moving average processes, when the original process is a sparse one.

Theorem 5. Consider an i.i.d. sparse process $Y$, such that $Y_{i} \sim p f_{c}+(1-p) \delta_{0}$, where $f_{c}$ denotes an absolutely continuous distribution with bounded support $(0,1)$. Define the causal moving average of process $Y$ as process $X$ defined as $X_{i}=\frac{1}{l} \sum_{j=1}^{l} Y_{i-j}$. Then,

$$
\bar{d}_{o}(X) \leq p
$$

\section{UNIVERSAL CS ALGORITHM}

Consider a stationary process $X=\left\{X_{i}\right\}_{i=1}^{\infty}$, such that $\bar{d}_{o}(X)<1$. As we argued in Section IV, since $\bar{d}_{o}(X)$ is strictly smaller than one, we expect this process to be structured. Therefore, intuitively, it might be possible to recover $X_{o}^{n}$ generated by source $X$ from an undersampled set of linear measurements $Y_{o}^{m}=A X_{o}^{n}, m<n$. In this section, we explore universal compressed sensing of such processes. We develop algorithms that are able to recover $X_{o}^{n}$ from enough linear measurements, without having any prior information about the source distribution. The proposed algorithms achieve the optimal performance for stationary memoryless sources with mixtures of discrete-continuous distributions, and therefore prove that, at least for such memoryless sources, there is no loss in the performance due to universal coding.

\section{A. Minimum entropy pursuit}

Consider the standard compressed sensing setup: instead of observing $X_{o}^{n}$, the decoder observes $Y_{o}^{m}=A X_{o}^{n}$, where $A \in \mathbb{R}^{m \times n}$ denotes the linear measurement matrix, and $m<n$. Further assume that the decoder does not have any knowledge about the distribution of the source. As we argued, for stationary processes $\bar{d}_{o}(X)$ measures the complexity of the source process. As a reminder, $\bar{d}_{o}(X)$ was defined as the limit of $\bar{d}_{k}(X)=$ $\limsup _{b} H\left(\left[X_{k+1}\right]_{b} \mid\left[X^{k}\right]_{b}\right) / b$. This suggests that, for the right choice of the parameters $b$ and $k, \hat{H}_{k}\left(\left[X^{n}\right]_{b}\right) / b$ defined as $H\left(U_{k+1} \mid U^{K}\right)$, where $U^{k+1} \sim p_{k+1}\left(\cdot \mid\left[X^{n}\right]_{b}\right)$, might serve as an estimator of $\bar{d}_{o}(X)$. Therefore, inspired by Occam's razor and this intuition, we propose minimum entropy pursuit (MEP) optimization, which recovers $X_{o}^{n}$ by solving the following optimization problem:

$$
\hat{X}_{o}^{n}=\underset{A x^{n}=Y_{o}^{m}}{\arg \min } \hat{H}_{k}\left(\left[x^{n}\right]_{b}\right),
$$

where $k$ and $b$ are parameters of the optimization. 
Note that MEP does not require knowledge of the source distribution and hence is a universal compressed sensing recovery algorithm. In the next section, we prove that if the stationary process $X$ satisfies certain constraints that will be specified later, and the parameters $k$ and $b$ are set appropriately, then the MEP optimization can reliably recover $X_{o}^{n}$ from measurements $Y^{m}$, as long as $m>(1+\delta) \bar{d}_{o}(X) n$, where $\delta>0$ can get arbitrary small.

\section{B. Theoretical analysis of MEP}

The main goal of this section is to show that MEP succeeds in recovering the source vector, without having access to its distribution. However, to prove this result, we have to impose a constraint on the source distribution. We first review this condition in Section V-B1, and then under these constraints, we characterize the performance of MEP in Section V-B2.

1) Mixing processes: To gain some insight on the constraint imposed on the input process, consider a simpler question. Suppose that the decoder has access to both the noiseless measurements $Y^{m}=A X_{o}^{n}$, and the complexity, or more specifically, the upper ID, of the process that has generated $X_{o}^{n}$. Now given a potential reconstruction sequence $\hat{x}^{n}$, is it possible to confirm whether $\hat{x}^{n}$ is a solution of the MEP optimization? Our heuristic answer to this question, may help the reader understand the constraints studied later. It is straightforward to check whether $\hat{x}^{n}$ satisfies the measurement constraints. Moreover, for $\hat{x}^{n}$ to be a solution of the MEP, in addition to satisfying the measurement equations, its complexity is expected to be close to the complexity of sequences generated by the source. For instance, if the decoder has access to a reliable estimator of $\bar{d}_{o}(X)$, it can apply it to $\hat{x}^{n}$, and for $n$ large enough, if $\hat{x}^{n}$ is equal or close to the source vector, it can expect the output to be close to $\bar{d}_{o}(X)$. The constraints imposed on process $X$ enables us to develop such sn estimator of $\bar{d}_{o}(X)$.

In the rest of the paper, we focus on $\psi^{*}$-mixing stationary processes. This condition ensures the convergence of our estimate of $\bar{d}_{o}(X)$. The $\psi^{*}$-mixing condition is a standard property studied in the ergodic theory literature. Consider a stationary process $X=\left\{X_{n}\right\}_{-\infty}^{\infty}$. Let $\mathcal{F}_{j}^{\ell}$ denote the $\sigma$-field of events generated by random variables $X_{j}^{k}$, where $j \leq k$. Define

$$
\psi^{*}(g)=\sup \frac{\mathrm{P}(\mathcal{A} \cap \mathcal{B})}{\mathrm{P}(\mathcal{A}) \mathrm{P}(\mathcal{B})}
$$

where the supremum is taken over all events $\mathcal{A} \in \mathcal{F}_{-\infty}^{j}$ and $\mathcal{B} \in \mathcal{F}_{j+g}^{\infty}$, where $P(\mathcal{A})>0$ and $\mathrm{P}(\mathcal{B})>0$.

Definition 3. A process $X=\left\{X_{n}\right\}_{-\infty}^{\infty}$ is called $\psi^{*}$-mixing, if $\psi^{*}(g) \rightarrow 1$, as g grows to infinity.

Intuitively, this condition ensures that the future and the past of the process that are well-separated are almost independent from each other. (For more information on $\psi^{*}$-mixing condition, and its connection to other mixing conditions, the reader is referred to [33].) In the following we review some $\psi^{*}$-mixing processes.

Example 1. Any i.i.d. process is $\psi^{*}$-mixing.

Example 2. All aperiodic Markov chains with finite sate space are $\psi^{*}$-mixing [31]. 
Example 3. Consider an i.i.d. process $Y=\left\{Y_{i}\right\}$ and define its moving average as $X_{i}=\frac{1}{l} \sum_{j=1}^{l} Y_{i-j}$. Then, process $X$ is $\Psi^{*}$-mixing.

Proof: Note that since process $Y$ is i.i.d. and since $l$ is finite, for $g$ large enough, $\mathcal{F}_{-\infty}^{j}$ and $\mathcal{F}_{j+g}^{\infty}$ are independent and therefore $\Psi^{*}(g)=1$.

In fact, by the same proof, the averaging function can be replaced by any fixed mapping $f: \mathcal{X}_{-l}^{l} \rightarrow \mathcal{X}$ and the process defined as $X_{i}=f\left(Y_{i-l}^{i+l}\right)$ is still $\Psi^{*}$-mixing.

Theorem III.1.7 of [31] proves that any $\Psi^{*}$ mixing process with finite alphabet has exponential rates of convergence for empirical distributions of all orders. The following theorem presents a straightforward extension of that result to $\Psi^{*}$-mixing processes with continuous alphabet. It proves that $b$-bit quantized versions of such processes have exponential rates for empirical frequencies of all orders, if $b$ is growing with $n$ slowly enough.

Theorem 6. Consider $\Psi^{*}$-mixing process $X=\left\{X_{i}\right\}$, with continuous alphabet $\mathcal{X}$. Let process $Z$ denote the b-bit quantized version of process $X$. That is, $Z=\left\{Z_{i}\right\}, Z_{i}=\left[X_{i}\right]_{b}$ and $\mathcal{Z}=\mathcal{X}_{b}$. Then, for any $\epsilon>0$, there exists $g \in \mathbb{N}$, depending only on $\epsilon$, such that for any $n>6(k+g) / \epsilon+k$,

$$
\mathrm{P}\left(\left\|p_{k}\left(\cdot \mid Z^{n}\right)-\mu_{k}\right\|_{1} \geq \epsilon\right) \leq 2^{c \epsilon^{2} / 8}(k+g) n^{|\mathcal{Z}|^{k}} 2^{\frac{-n c \epsilon^{2}}{8(k+g)}},
$$

where $c=1 /(2 \ln 2)$. Here, for $a^{k} \in \mathcal{Z}^{k}, \mu_{k}\left(a^{k}\right)=\mathrm{P}\left(Z^{k}=a^{k}\right)$

Proof: The proof is excatly as the proof of Theorem III.1.7 of [31]. The shift from continuous-alphabet sources to finite-alphabet sources is done by the quantization of the source and by also noting that if a continuous-alphabet process is $\Psi^{*}$-mixing, its quantized version is also $\Psi^{*}$-mixing. To see this, for $j \leq k$, let $\mathcal{F}_{j}^{k}$ and $\hat{\mathcal{F}}_{j}^{k}$ denote the sigma-fields generated by $X_{j}^{k}$ and $Z_{j}^{k}$, respectively. But since $\hat{\mathcal{F}}_{j}^{k}$ is always a sub sigma-field of $\mathcal{F}_{j}^{k}$, we have

$$
\begin{aligned}
\psi_{Z}^{*}(g) & =\sup _{\mathcal{A} \in \hat{\mathcal{F}}_{-\infty}^{j}, \mathcal{B} \in \hat{\mathcal{F}}_{j+g}^{\infty}} \frac{\mathrm{P}(\mathcal{A} \cap \mathcal{B})}{\mathrm{P}(\mathcal{A}) \mathrm{P}(\mathcal{B})} \\
& \leq \sup _{\mathcal{A} \in \mathcal{F}_{-\infty}^{j}, \mathcal{B} \in \mathcal{F}_{j+g}^{\infty}} \frac{\mathrm{P}(\mathcal{A} \cap \mathcal{B})}{\mathrm{P}(\mathcal{A}) \mathrm{P}(\mathcal{B})} \\
& =\Psi_{X}^{*}(g) .
\end{aligned}
$$

But since the continuous process is known to be $\Psi^{*}$-mixing, $\Psi_{X}^{*}(g)$ converges to one, as $g$ grows to infinity. This proves that process $Z$ is also $\Psi^{*}$-mixing, with a $\Psi^{*}(g)$ function than is upper-bounded with that of $\Psi_{X}^{*}(g)$.

2) Performance of MEP: The following theorem proves that MEP is a universal decoder for $\Psi^{*}$-mixing processes.

Theorem 7. Consider a $\Psi^{*}$-mixing stationary process $\left\{X_{i}\right\}_{i=1}^{\infty}$, with $\mathcal{X}=[0,1]$ and upper information dimension $\bar{d}_{o}(X)$. Let $b=b_{n}=\lceil\log \log n\rceil, k=k_{n}=o\left(\frac{\log n}{\log \log n}\right)$ and $m=m_{n} \geq(1+\delta) \bar{d}_{o}(X) n$, where $\delta>0$. For each $n$, let the entries of the measurement matrix $A=A_{n} \in \mathbb{R}^{m \times n}$ be drawn i.i.d. according to $\mathcal{N}(0,1)$. For $X_{o}^{n}$ generated by the source $X$ and $Y_{o}^{m}=A X_{o}^{n}$, let $\hat{X}_{o}^{n}=\hat{X}_{o}^{n}\left(Y_{o}^{m}, A\right)$ denote the solution of (3), i.e., 
$\hat{X}_{o}^{n}=\arg \min _{A x^{n}=Y_{o}^{m}} \hat{H}_{k}\left(\left[x^{n}\right]_{b}\right)$. Then,

$$
\frac{1}{\sqrt{n}}\left\|X_{o}^{n}-\hat{X}_{o}^{n}\right\|_{2} \stackrel{\mathrm{P}}{\longrightarrow} 0 .
$$

Remark 1. Theorem 7 proves that, in the asymptotic setting, as the blocklength $n$ grows to infinity, the normalized number of measurements $(\mathrm{m} / \mathrm{n})$ required by $M E P$ for recovering memoryless sources with discrete-continuous mixture distributions coincides with the fundamental limits of non-universal compressed sensing characterized in [30]. In other words, this result shows that, at least for such sources, there is no loss in performance due to universality. This proves that, asymptotically, at least for such stationary memoryless sources, similar to data compression, denoising, and prediction, there is no loss in universal compressed sensing, due to not knowing the source distribution.

The optimization presented in (3) is not easy to handle. While the search domain, i.e., the set of points satisfying $A x^{n}=y_{o}^{m}$, is a hyperplane, the cost function is defined on a discretized space, which is formed by the quantized version of the source alphabet $\mathcal{X}$. To move towards designing an implementable universal compressed sensing algorithm, consider the following Lagrangian-type approximation of MEP:

$$
\hat{x}_{o}^{n}=\underset{u^{n} \in \mathcal{X}_{b}^{n}}{\arg \min }\left(\hat{H}_{k}\left(u^{n}\right)+\frac{\lambda}{n^{2}}\left\|A u^{n}-y_{o}^{m}\right\|_{2}^{2}\right),
$$

where $\mathcal{X}_{b} \triangleq\left\{[x]_{b}: x \in \mathcal{X}\right\}$. We refer to this algorithm as Lagrangian-MEP. The main difference between (3) and (5) is that in (5) the search space is now a discrete set. The advantage of Lagrangian-MEP compared to MEP is that it is implementable and classic discrete optimization methods such as Markov chain Monte Carlo (MCMC) and simulated annealing [34]-[36] can be employed to approximate its optimizer.

The Lagrangian-MEP algorithm is in fact identical to the heuristic algorithm for universal compressed sensing proposed in [21] and [22]. In [21] and [22], Baron et al. employ simulated annealing and Markov chain Monte Carlo techniques to approximate the minimizer of the Lagrangian-MEP cost function. The following theorem shows that for the right choice of parameter $\lambda,(5)$ is in fact a universal compressed sensing algorithm, which approximates the solution of MEP with no asymptotic loss in performance.

Theorem 8. Consider a $\Psi^{*}$-mixing stationary process $\left\{X_{i}\right\}_{i=1}^{\infty}$, with $\mathcal{X}=[0,1]$ and upper information dimension $\bar{d}_{o}(X)$. Let $b=b_{n}=\lceil r \log \log n\rceil$, where $r>1, k=k_{n}=o\left(\frac{\log n}{\log \log n}\right), \lambda=\lambda_{n}=(\log n)^{2 r}$ and $m=m_{n} \geq$ $(1+\delta) \bar{d}_{o}(X) n$, where $\delta>0$. For each $n$, let the entries of the measurement matrix $A=A_{n} \in \mathbb{R}^{m \times n}$ be drawn i.i.d. according to $\mathcal{N}(0,1)$. Given $X_{o}^{n}$ generated by source $X$ and $Y_{o}^{m}=A X_{o}^{n}$, let $\hat{X}_{o}^{n}=\hat{X}_{o}^{n}\left(Y_{o}^{m}, A\right)$ denote the solution of (5), i.e., $\hat{X}_{o}^{n}=\arg \min _{u^{n} \in \mathcal{X}^{n}}\left(\hat{H}_{k}\left(u^{n}\right)+\frac{\lambda}{n^{2}}\left\|A u^{n}-Y_{o}^{m}\right\|_{2}^{2}\right)$. Then,

$$
\frac{1}{\sqrt{n}}\left\|X_{o}^{n}-\hat{X}_{o}^{n}\right\|_{2} \stackrel{\mathrm{P}}{\longrightarrow} 0 \text {. }
$$

So far we assumed that the measurements are perfect and noise-free. In almost all practical situations the measurements are contaminated by noise. Therefore, it is important to study the performance of the proposed 
algorithms in the presence of noise. We next prove that the Lagrangian-MEP algorithm, which was proved to be an implementable universal compressed sensing algorithm, is also robust to measurement noise.

Assume that instead of $A x_{o}^{n}$, the decoder observes $y_{o}^{m}=A x_{o}^{n}+z^{m}$, where $z^{m}$ denotes the noise in the measurement system, and employs the Lagrangian-MEP to recover $x_{o}^{n}$, i.e.,

$$
\hat{x}^{n}=\underset{u^{n} \in \mathcal{X}_{b}^{n}}{\arg \min }\left(\hat{H}_{k}\left(u^{n}\right)+\frac{\lambda}{n^{n}}\left\|A u^{n}-y_{o}^{m}\right\|_{2}\right) .
$$

The following theorem proves that the Lagrangian-MEP is robust to measurement noise, and as long as the $\ell_{2}$ norm of the noise vector is small enough, the algorithm recovers the source vector from the same number of measurements, despite receiving noisy observations.

Theorem 9. Consider a $\Psi^{*}$-mixing stationary process $\left\{X_{i}\right\}_{i=1}^{\infty}$, with $\mathcal{X}=[0,1]$ and upper information dimension $\bar{d}_{o}(X)$. Consider a measurement matrix $A=A_{n} \in \mathbb{R}^{m \times n}$ with i.i.d. entries distributed according to $\mathcal{N}(0,1)$. Let $b=b_{n}=\lceil r \log \log n\rceil$, where $r>1, k=k_{n}=o\left(\frac{\log n}{\log \log n}\right), \lambda=\lambda_{n}=(\log n)^{2 r}$ and $m=m_{n} \geq(1+\delta) \bar{d}_{o}(X) n$, where $\delta>0$. For $X_{o}^{n}$ generated by the source $X$, we observe $Y_{o}^{m}=A X_{o}^{n}+Z^{m}$, where $Z^{m}$ denotes the measurement noise. Assume that there exists a deterministic sequence $c_{m}$ such that $\lim _{m \rightarrow \infty} \mathrm{P}\left(\left\|Z^{m}\right\|_{2}>c_{m}\right)=0$, and $c_{m}=O\left(m /(\log m)^{r}\right)$. Let $\hat{X}_{o}^{n}=\hat{X}_{o}^{n}\left(Y_{o}^{m}, A\right)$ denote the solution of (5). Then, $\frac{1}{\sqrt{n}}\left\|X_{o}^{n}-\hat{X}_{o}^{n}\right\|_{2} \stackrel{\mathrm{P}}{\longrightarrow} 0$.

\section{PROOFS}

Before presenting the proofs of the results, we state two useful lemmas that are used later in the proofs. The first lemma in the following is from [25].

Lemma 4 ( $\chi^{2}$ concentration). Fix $\tau>0$, and let $U_{i} \stackrel{\text { i.i.j. }}{\sim} \mathcal{N}(0,1), i=1,2, \ldots, m$. Then,

$$
\mathrm{P}\left(\sum_{i=1}^{m} U_{i}^{2}<m(1-\tau)\right) \leq \mathrm{e}^{\frac{m}{2}(\tau+\ln (1-\tau))}
$$

and

$$
\mathrm{P}\left(\sum_{i=1}^{m} U_{i}^{2}>m(1+\tau)\right) \leq \mathrm{e}^{-\frac{m}{2}(\tau-\ln (1+\tau))}
$$

Lemma 5. Consider distributions $p$ and $q$ on finite alphabet $\mathcal{X}$ such that $\|p-q\|_{1} \leq \epsilon$. Then,

$$
|H(p)-H(q)| \leq-\epsilon \log \epsilon+\epsilon \log |\mathcal{X}|
$$

Proof: Define $f(y)=-y \ln y$, for $y \in[0,1]$, and $g(y)=f(y+\epsilon)-f(y)$. Since $g^{\prime}(y)=\ln (y /(y+\epsilon))<0, g$ is a decreasing function of $y$. Therefore,

$$
g(y) \leq-\epsilon \ln \epsilon .
$$


For $x \in \mathcal{X}$, let $|p(x)-q(x)|=\epsilon_{x}$. By our assumption,

$$
\sigma \triangleq \sum_{x \in \mathcal{X}} \epsilon_{x} \leq \epsilon
$$

On the other hand, we just proved that

$$
|-p(x) \ln p(x)+q(x) \ln q(x)| \leq-\epsilon_{x} \ln \epsilon_{x}
$$

Therefore,

$$
\begin{aligned}
|H(p)-H(q)| & =\left|\sum_{x \in \mathcal{X}}(-p(x) \ln p(x)+q(x) \ln q(x))\right| \\
& \leq \sum_{x \in \mathcal{X}}|-p(x) \ln p(x)+q(x) \ln q(x)| \\
& \leq \sum_{x \in \mathcal{X}}-\epsilon_{x} \ln \epsilon_{x} .
\end{aligned}
$$

Also

$$
\begin{aligned}
\sum_{x \in \mathcal{X}}-\epsilon_{x} \log \epsilon_{x} & =\sum_{x \in \mathcal{X}}-\epsilon_{x} \log \frac{\epsilon_{x} \sigma}{\sigma} \\
& =-\sigma \log \sigma+\sigma H\left(\frac{\epsilon_{x}}{\sigma}: x \in \mathcal{X}\right) \\
& \leq-\epsilon \log \epsilon+\epsilon \log |\mathcal{X}|
\end{aligned}
$$

where the last line follows because $f(y)$ is an increasing function for $y \leq \mathrm{e}^{-1}$.

\section{A. Proof of Lemma 3}

Since the process is stationary,

$$
\begin{aligned}
H\left(\left[X^{k}\right]_{b}\right) & =\sum_{i=1}^{k} H\left(\left[X_{i}\right]_{b} \mid\left[X^{i-1}\right]_{b}\right) \\
& =\sum_{i=1}^{k} H\left(\left[X_{k}\right]_{b} \mid\left[X_{k-i+1}^{k-1}\right]_{b}\right) \\
& \geq k H\left(\left[X_{k}\right]_{b} \mid\left[X^{k-1}\right]_{b}\right) .
\end{aligned}
$$

Therefore,

$$
\begin{aligned}
\frac{1}{k}\left(\limsup _{b \rightarrow \infty} \frac{H\left(\left[X^{k}\right]_{b}\right)}{b}\right) & \geq \limsup _{b \rightarrow \infty} \frac{H\left(\left[X_{k}\right]_{b} \mid\left[X^{k-1}\right]_{b}\right)}{b} \\
& =\bar{d}_{k}(X)
\end{aligned}
$$


Taking liminf of both as $k$ grows to infinity proves that

$$
\liminf _{k \rightarrow \infty} \frac{1}{k}\left(\limsup _{b \rightarrow \infty} \frac{H\left(\left[X^{k}\right]_{b}\right)}{b}\right) \geq \bar{d}_{o}(X)
$$

On the other hand, for any set of functions $f_{1}, \ldots, f_{k}$, and any $B \in \mathbb{R}, \sup _{b>b_{o}} \sum_{i=1}^{k} f_{i}(b) \leq \sum_{i=1}^{k} \sup _{b>b_{o}} f_{i}(b)$. Taking the limit of both sides as $b_{o}$ grows to infinity yields $\lim _{\sup _{b}} \sum_{i=1}^{k} f_{i}(b) \leq \sum_{i=1}^{k} \limsup _{b} f_{i}(b)$. Therefore, letting $f_{i}(b)=b^{-1} H\left(\left[X_{k}\right]_{b} \mid\left[X_{k-i+1}^{k-1}\right]_{b}\right)$, it follows that

$$
\begin{aligned}
\limsup _{b \rightarrow \infty} \frac{H\left(\left[X^{k}\right]_{b}\right)}{b k} & =\limsup _{b \rightarrow \infty} \frac{\sum_{i=1}^{k} H\left(\left[X_{k}\right]_{b} \mid\left[X_{k-i+1}^{k-1}\right]_{b}\right)}{b k} \\
& \leq \frac{1}{k} \sum_{i=1}^{k} \limsup _{b \rightarrow \infty} \frac{H\left(\left[X_{k}\right]_{b} \mid\left[X_{k-i+1}^{k-1}\right]_{b}\right)}{b} \\
& =\frac{1}{k} \sum_{i=0}^{k-1} \bar{d}_{i}(X) .
\end{aligned}
$$

For a sequence of numbers $\left(a_{k}\right)_{k}$, such that $\lim _{k \rightarrow \infty} a_{k}=a$, the Cesàro mean theorem states that $b_{k}=\frac{1}{k} \sum_{i=1}^{k} a_{i}$ also converges to $a$. Therefore, since $\lim _{i \rightarrow \infty} \bar{d}_{i}(X)=\bar{d}_{o}(X)$, by the Cesàro mean theorem, we have

$$
\lim _{k \rightarrow \infty} \frac{1}{k} \sum_{i=0}^{k-1} \bar{d}_{i}(X)=\bar{d}_{o}(X) .
$$

Taking the limsup of both sides of (12) yields

$$
\limsup _{k \rightarrow \infty} \limsup _{b \rightarrow \infty} \frac{H\left(\left[X^{k}\right]_{b}\right)}{b k} \leq \bar{d}_{o}(X) .
$$

The desired result follows from combining (11) and (13).

\section{B. Proof of Theorem 3}

We first show that the quantized versions of $X$ at any quantization level $b$ is also a stationary first-order Markov process. To show this, let $Z_{k}$ denote the i.i.d. Bernoulli process that indicates the positions of the jumps in process $X$. In other words, $Z_{k}=\mathbb{1}_{X_{k} \neq X_{k-1}}$. Then, for any $u^{k+1} \in \mathcal{X}_{b}^{k+1}$, we have

$$
\begin{aligned}
\mathrm{P}\left(\left[X_{k+1}\right]_{b}=u_{k+1} \mid\left[X^{k}\right]_{b}=u^{k}\right)= & \mathrm{P}\left(\left[X_{k+1}\right]_{b}=u_{k+1}, Z_{k+1}=0 \mid\left[X^{k}\right]_{b}=u^{k}\right) \\
& +\mathrm{P}\left(\left[X_{k+1}\right]_{b}=u_{k+1}, Z_{k+1}=1 \mid\left[X^{k}\right]_{b}=u^{k}\right) .
\end{aligned}
$$

But, since by the definition of process $X, Z_{k+1}$ is independent of $X^{k}$,

$$
\begin{aligned}
\mathrm{P}\left(\left[X_{k+1}\right]_{b}=u_{k+1}, Z_{k+1}=0 \mid\left[X^{k}\right]_{b}=u^{k}\right) & =\mathrm{P}\left(Z_{k+1}=0 \mid\left[X^{k}\right]_{b}=u^{k}\right) \mathrm{P}\left(\left[X_{k+1}\right]_{b}=u_{k+1} \mid Z_{k+1}=0,\left[X^{k}\right]_{b}=u^{k}\right) \\
& =(1-p) \mathrm{P}\left(\left[X_{k+1}\right]_{b}=u_{k+1}\right) .
\end{aligned}
$$


and

$$
\begin{gathered}
\mathrm{P}\left(\left[X_{k+1}\right]_{b}=u_{k+1}, Z_{k+1}=1 \mid\left[X^{k}\right]_{b}=u^{k}\right) \\
=\mathrm{P}\left(\left[X_{k+1}\right]_{b}=\mathrm{P}\left(Z_{k+1}=1 \mid\left[X^{k}\right]_{b}=u^{k}\right) u_{k+1} \mid Z_{k+1}=1,\left[X^{k}\right]_{b}=u^{k}\right) \\
=p \mathbb{1}_{u_{k+1}=u_{k}}
\end{gathered}
$$

Therefore, overall,

$$
\mathrm{P}\left(\left[X_{k+1}\right]_{b}=u_{k+1} \mid\left[X^{k}\right]_{b}=u^{k}\right)=(1-p) \mathrm{P}\left(\left[X_{k+1}\right]_{b}=u_{k+1}\right)+p \mathbb{1}_{u_{k+1}=u_{k}},
$$

which only depends on $u_{k}$. Therefore $[X]_{b}$ is also a first-order Markov process. Stationarity of $[X]_{b}$ follows immediately.

Now since the quantized process is also a stationary first-order Markov process,

$$
H\left(\left[X_{k+1}\right]_{b} \mid\left[X^{k}\right]_{b}\right)=H\left(\left[X_{k+1}\right]_{b} \mid\left[X_{k}\right]_{b}\right)=H\left(\left[X_{2}\right]_{b} \mid\left[X_{1}\right]_{b}\right)
$$

Therefore,

$$
\bar{d}_{k}(X)=\bar{d}_{1}(X)
$$

and

$$
\underline{d}_{k}(X)=\underline{d}_{1}(X)
$$

for all $k \geq 1$. Let $\mathcal{X}_{b}=\left\{[x]_{b}: x \in \mathcal{X}\right\}$ denote the alphabet at resolution $b$. Then,

$$
H\left(\left[X_{2}\right]_{b} \mid\left[X_{1}\right]_{b}\right)=\sum_{c \in \mathcal{X}_{b}} \mathrm{P}\left(\left[X_{1}\right]_{b}=c\right) H\left(\left[X_{2}\right]_{b} \mid\left[X_{1}\right]_{b}=c\right) .
$$

We next prove that $\frac{1}{b} H\left(\left[X_{2}\right]_{b} \mid\left[X_{1}\right]_{b}=c_{1}\right)$ uniformly converges to $p$, as $b$ grows to infinity, for all values of $c_{1}$.

Define the indicator random variable $I=\mathbb{1}_{X_{2}=X_{1}}$. Given the transition probability of the Markov chain, $I$ is independent of $X_{1}$, and $\mathrm{P}(I=1)=1-p$. Also define a random variable $U$, independent of $\left(X_{1}, X_{2}\right)$, and distributed according to $f_{c}$. Then, it follows that

$$
\begin{aligned}
\mathrm{P}\left(\left[X_{2}\right]_{b}=c_{2} \mid\left[X_{1}\right]_{b}=c_{1}\right)= & \mathrm{P}\left(\left[X_{2}\right]_{b}=c_{2}, I=0 \mid\left[X_{1}\right]_{b}=c_{1}\right) \\
& +\mathrm{P}\left(\left[X_{2}\right]_{b}=c_{2}, I=1 \mid\left[X_{1}\right]_{b}=c_{1}\right) \\
= & p \mathrm{P}\left(\left[X_{2}\right]_{b}=c_{2} \mid\left[X_{1}\right]_{b}=c_{1}, I=0\right) \\
& +(1-p) \mathrm{P}\left(\left[X_{2}\right]_{b}=c_{2} \mid\left[X_{1}\right]_{b}=c_{1}, I=1\right) \\
= & p \mathrm{P}\left([U]_{b}=c_{2}\right)+(1-p) \mathbb{1}_{c_{2}=c_{1}},
\end{aligned}
$$

where the last line follows from the fact that conditioned on $X_{2} \neq X_{1}, X_{2}$, independent of the value of $X_{1}$, is 
distributed according to $f_{c}$. For $a \in \mathcal{X}_{b}$,

$$
P\left([U]_{b}=a\right)=\int_{a}^{a+2^{-b}} f_{c}(u) d u .
$$

On the other hand, by the mean value theorem, there exists $x_{a} \in\left[a, a+2^{-b}\right]$, such that

$$
2^{b} \int_{a}^{a+2^{-b}} f_{c}(u) d u=f_{c}\left(x_{a}\right) .
$$

Therefore, $P\left([U]_{b}=a\right)=2^{-b} f_{c}\left(x_{a}\right)$, and

$$
\begin{aligned}
H\left(\left[X_{2}\right]_{b} \mid\left[X_{1}\right]_{b}=c_{1}\right)= & -\sum_{a \in \mathcal{X}_{b}, a \neq c_{1}}-\left(p 2^{-b} f_{c}\left(x_{a}\right)\right) \log \left(p 2^{-b} f_{c}\left(x_{a}\right)\right) \\
& -\left(p 2^{-b} f_{c}\left(x_{c_{1}}\right)+1-p\right) \log \left(p 2^{-b} f_{c}\left(x_{c_{1}}\right)+1-p\right) \\
= & \sum_{a \in \mathcal{X}_{b}, a \neq c_{1}}-\left(p 2^{-b} f_{c}\left(x_{a}\right)\right)\left(\log p-b+\log \left(f_{c}\left(x_{a}\right)\right)\right) \\
& -\left(p 2^{-b} f_{c}\left(x_{c_{1}}\right)+1-p\right) \log \left(p 2^{-b} f_{c}\left(x_{c_{1}}\right)+1-p\right) .
\end{aligned}
$$

Dividing both sides of (15) by $b$ yields

$$
\begin{aligned}
\frac{H\left(\left[X_{2}\right]_{b} \mid\left[X_{1}\right]_{b}=c_{1}\right)}{b}= & \frac{(b-\log p) p}{b} \sum_{a \in \mathcal{X}_{b}, a \neq c_{1}} 2^{-b} f_{c}\left(x_{a}\right) \\
& -\left(\frac{p}{b}\right) \sum_{a \in \mathcal{X}_{b}, a \neq c_{1}} 2^{-b} f_{c}\left(x_{a}\right) \log \left(f_{c}\left(x_{a}\right)\right) \\
& -\frac{\left(p 2^{-b} f_{c}\left(x_{c_{1}}\right)+1-p\right) \log \left(p 2^{-b} f_{c}\left(x_{c_{1}}\right)+1-p\right)}{b} .
\end{aligned}
$$

On the other hand, from (14),

$$
\begin{aligned}
\sum_{a \in \mathcal{X}_{b}} 2^{-b} f_{c}\left(x_{a}\right) & =\sum_{a \in \mathcal{X}_{b}} \int_{a}^{a+2^{-b}} f_{c}(u) d u \\
& =\int_{0}^{1} f_{c}(u) d u \\
& =1 .
\end{aligned}
$$

Also, since $\int_{0}^{1} f_{c}(u) d u=1$,

$$
\lim _{b \rightarrow \infty} \sum_{a \in \mathcal{X}_{b}} 2^{-b} f_{c}\left(x_{a}\right) \log \left(f_{c}\left(x_{a}\right)\right)=h\left(f_{c}\right),
$$

where $h\left(f_{c}\right)=-\int f_{c}(u) \log f_{c}(u) d u$ denotes the differential entropy of $U$. Therefore, for any $\epsilon>0$, there exists $b_{\epsilon} \in \mathbb{N}$, such that for $b>b_{\epsilon}$,

$$
\left|\sum_{a \in \mathcal{X}_{b}} 2^{-b} f_{c}\left(x_{a}\right) \log \left(f_{c}\left(x_{a}\right)\right)-h\left(f_{c}\right)\right| \leq \epsilon .
$$


Since $f_{c}$ is bounded by assumption, $M=\sup _{x \in[0,1]} f_{c}(x)<\infty$, and $h\left(f_{c}\right) \leq \log M<\infty$. Finally, $-q \log q \leq$ $\mathrm{e}^{-1} \log \mathrm{e}$, for $q \in[0,1]$. Therefore, combining (16), (17) and (18), it follows that, for $b>b_{\epsilon}$,

$$
\left|\frac{H\left(\left[X_{2}\right]_{b} \mid\left[X_{1}\right]_{b}=c_{1}\right)}{b}-p\right| \leq \frac{M}{2^{b}}-\frac{p \log p}{b}+\frac{p\left(h\left(f_{c}\right)+\epsilon\right)}{b}+\frac{\log \mathrm{e}}{\mathrm{e} b},
$$

for all $c_{1} \in \mathcal{X}_{b}$. Since the right hand side of the above equation does not depend on $c_{1}$, and goes to zero as $b \rightarrow \infty$, for any $\epsilon^{\prime}>0$, there exists $b_{\epsilon^{\prime}}$, such that for $b>\max \left\{b_{\epsilon}, b_{\epsilon^{\prime}}\right\}$,

$$
\left|\frac{H\left(\left[X_{2}\right]_{b} \mid\left[X_{1}\right]_{b}=c_{1}\right)}{b}-p\right| \leq \epsilon^{\prime}
$$

and

$$
\begin{aligned}
\left|\frac{H\left(\left[X_{2}\right]_{b} \mid\left[X_{1}\right]_{b}\right)}{b}-p\right| & \leq \sum_{c_{1} \in \mathcal{X}_{b}} \mathrm{P}\left(\left[X_{1}\right]_{b}=c_{1}\right)\left|\frac{H\left(\left[X_{2}\right]_{b} \mid\left[X_{1}\right]_{b}=c_{1}\right)}{b}-p\right| \\
& \leq \epsilon^{\prime} \sum_{c_{1} \in \mathcal{X}_{b}} \mathrm{P}\left(\left[X_{1}\right]_{b}=c_{1}\right) \\
& =\epsilon^{\prime}
\end{aligned}
$$

which concludes the proof.

\section{Proof of Theorem 4}

Since the process is stationary and Markov of order $l$, by Theorem $2 \lim \sup _{b \rightarrow \infty} b^{-1} H\left(\left[X_{l+1}\right]_{b} \mid X^{l}\right) \leq \bar{d}_{o}(X) \leq$ $\bar{d}_{\ell}(X)$ and $\liminf _{b \rightarrow \infty} b^{-1} H\left(\left[X_{l+1}\right]_{b} \mid X^{l}\right) \leq \underline{d}_{o}(X) \leq \underline{d}_{\ell}(X)$.

Define the indicator random variable $I=\mathbb{1}_{Z_{l}=0}$. By the definition of the Markov chain, $\mathrm{P}(I=1)=1-p$. Then, since $I$ is independent of $X^{l}$, we have

$$
\begin{aligned}
H\left(\left[X_{l+1}\right]_{b} \mid\left[X^{l}\right]_{b}\right) \leq & H\left(\left[X_{l+1}\right]_{b}, I \mid\left[X^{l}\right]_{b}\right) \\
\leq & 1+H\left(\left[X_{l+1}\right]_{b} \mid\left[X^{l}\right]_{b}, I\right) \\
= & 1+p H\left(\left[\sum_{i=1}^{l} a_{i} X_{l-i}+U\right]_{b} \mid\left[X^{l}\right]_{b}\right) \\
& +(1-p) H\left(\left[\sum_{i=1}^{l} a_{i} X_{l-i}\right]_{b} \mid\left[X^{l}\right]_{b}\right),
\end{aligned}
$$

where $U$ is independent of $X^{l}$ and is distributed according to $f_{c}$.

Conditioned on $\left[X^{l}\right]_{b}=c^{l}$, where $c_{1}, \ldots, c_{l+1} \in \mathcal{X}_{b}$, we have

$$
c_{i} \leq X_{i}<c_{i}+2^{-b},
$$

for $i=1, \ldots, l$, and

$$
\left|\sum_{i=1}^{l} a_{i} X_{l-i}-\sum_{i=1}^{l} a_{i} c_{l-i}\right| \leq 2^{-b} \sum_{i=1}^{l}\left|a_{i}\right| .
$$


Let $M=\left\lceil\sum_{i=1}^{l}\left|a_{i}\right|\right\rceil$ and $c=\sum_{i=1}^{l} a_{i} c_{l-i}$. Then,

$$
\sum_{i=1}^{l} a_{i} X_{l-i} \in\left[c-M 2^{-b}, c+M 2^{-b}\right]
$$

Therefore, $\left[\sum_{i=1}^{l} a_{i} X_{l-i}\right]_{b}$ can take only $2 M+1$ different values, and as a result $H\left(\left[\sum_{i=1}^{l} a_{i} X_{l-i}\right]_{b} \mid\left[X^{l}\right]_{b}\right) \leq$ $\log (2 M+1)$. Since $M$ does not depend on $b$, it follows that

$$
\lim _{b \rightarrow \infty} \frac{H\left(\left[\sum_{i=1}^{l} a_{i} X_{l-i}\right]_{b} \mid\left[X^{l}\right]_{b}\right)}{b}=0 .
$$

We next prove that

$$
\lim _{b \rightarrow \infty} \frac{H\left(\left[\sum_{i=1}^{l} a_{i} X_{l-i}+U\right]_{b} \mid\left[X^{l}\right]_{b}\right)}{b}=1
$$

for any absolutely continuous distribution with pdf $f_{c}$. This proves that $\bar{d}_{l}(X)=\underline{d}_{l}(X)=p$.

To bound $H\left(\left[\sum_{i=1}^{l} a_{i} X_{l-i}+U\right]_{b} \mid\left[X^{l}\right]_{b}\right)$, we need to study $\mathrm{P}\left(\left[\sum_{i=1}^{l} a_{i} X_{l-i}+U\right]_{b}=c \mid\left[X^{l}\right]_{b}=c^{l}\right)$, where $c^{l} \in \mathcal{X}_{b}^{l}$, and $c \in \mathcal{X}_{b}$. Let $\mathcal{N}\left(c^{l}, b\right)=\left\{x^{l}: c_{i} \leq x_{i} \leq c_{i}+2^{-b}, i=1, \ldots, l\right\}$, and define the function $g: \mathbb{R}^{l} \rightarrow \mathbb{R}$, by $g\left(x^{l}\right)=\sum_{i=1}^{l} a_{i} x_{l-i}$. Note that

$$
\begin{aligned}
\mathrm{P}\left(\left[\sum_{i=1}^{l} a_{i} X_{l-i}+U\right]_{b}=c \mid\left[X^{l}\right]_{b}=c^{l}\right) & =\frac{\mathrm{P}\left(\left[\sum_{i=1}^{l} a_{i} X_{l-i}+U\right]_{b},\left[X^{l}\right]_{b}=c^{l}\right)}{\mathrm{P}\left(\left[X^{l}\right]_{b}=c^{l}\right)} \\
& =\frac{\int_{\mathcal{N}\left(c^{l}, b\right)} \int_{c-g\left(x^{l}\right)}^{c-g\left(x^{l}\right)+2^{-b}} f\left(x^{l}\right) f_{c}(u) d u d x^{l}}{\mathrm{P}\left(\left[X^{l}\right]_{b}=c^{l}\right)}
\end{aligned}
$$

where $f\left(x^{l}\right)$ denotes the pdf of $X^{l}$. By the mean value theorem, there exists $\delta\left(x^{l}\right) \in\left(0,2^{-b}\right)$, such that

$$
\int_{c-g\left(x^{l}\right)}^{c-g\left(x^{l}\right)+2^{-b}} f_{c}(u) d u=2^{-b} f_{c}\left(c-g\left(x^{l}\right)+\delta\left(x^{l}\right)\right) .
$$

Combining (22) and (23) yields that

$$
\mathrm{P}\left(\left[\sum_{i=1}^{l} a_{i} X_{l-i}+U\right]_{b}=c \mid\left[X^{l}\right]_{b}=c^{l}\right)=\frac{2^{-b} \int_{\mathcal{N}\left(c^{l}, b\right)} f\left(x^{l}\right) f_{c}\left(c-g\left(x^{l}\right)+\delta\left(x^{l}\right)\right) d x^{l}}{\int_{\mathcal{N}\left(c^{l}, b\right)} f\left(x^{l}\right) d x^{l}} .
$$

Define the pdf $p_{c^{l}, b}\left(y^{l}\right)$ over $\mathcal{N}\left(c^{l}, b\right)$ as

$$
p_{c^{l}, b}\left(y^{l}\right)=\frac{f\left(y^{l}\right)}{\int_{\mathcal{N}\left(c^{l}, b\right)} f\left(x^{l}\right) d x^{l}} .
$$


Then, $\mathrm{P}\left(\left[\sum_{i=1}^{l} a_{i} X_{l-i}+U\right]_{b}=c \mid\left[X^{l}\right]_{b}=c^{l}\right)=2^{-b} \mathrm{E}\left[f_{c}\left(c-g\left(Y^{l}\right)-\delta\left(Y^{l}\right)\right)\right]$, where $Y^{l} \sim p_{c^{l}, b}$. Hence,

$$
\begin{aligned}
& H\left(\left[\sum_{i=1}^{l} a_{i} X_{l-i}+U\right]_{b} \mid\left[X^{l}\right]_{b}\right)=\sum_{c^{l}} \sum_{c}\left(b-\log \mathrm{E}\left[f_{c}\left(c-g\left(Y^{l}\right)-\delta\left(Y^{l}\right)\right)\right]\right) \\
& \times \mathrm{P}\left(\left[\sum_{i=1}^{l} a_{i} X_{l-i}+U\right]_{b}=c \mid\left[X^{l}\right]_{b}=c^{l}\right) \\
&=b-\sum_{c^{l}} \sum_{c} \log \mathrm{E}\left[f_{c}\left(c-g\left(Y^{l}\right)-\delta\left(Y^{l}\right)\right)\right] \\
& \times \mathrm{P}\left(\left[\sum_{i=1}^{l} a_{i} X_{l-i}+U\right]_{b}=c \mid\left[X^{l}\right]_{b}=c^{l}\right) .
\end{aligned}
$$

Since by assumption $f_{c}$ is bounded on its support between $\alpha$ and $\beta$, then for $b$ large enough, if $c-\sum_{i=1}^{l} a_{i} c_{l-i} \in \mathcal{Z}$, then $\mathrm{E}\left[f_{c}\left(c-g\left(Y^{l}\right)-\delta\left(Y^{l}\right)\right)\right]$ is also bounded between $\alpha$ and $\beta$, and hence the desired result follows. That is, $\lim _{b \rightarrow \infty} b^{-1} H\left(\left[\sum_{i=1}^{l} a_{i} X_{l-i}+U\right]_{b} \mid\left[X^{l}\right]_{b}\right)=1$.

For the lower bound, we next prove that $\limsup _{b \rightarrow \infty} \frac{H\left(\left[X_{l+1}\right]_{b} \mid X^{l}\right)}{b} \geq p$ and $\liminf _{b \rightarrow \infty} \frac{H\left(\left[X_{l+1}\right]_{b} \mid X^{l}\right)}{b} \geq p$. Note that

$$
\begin{aligned}
H\left(\left[X_{l+1}\right]_{b} \mid X^{l}\right) & \geq H\left(\left[X_{l+1}\right]_{b} \mid X^{l}, I\right) \\
& =p H\left(\left[\sum_{i=1}^{l} a_{i} X_{l-i}+U\right]_{b} \mid X^{l}\right)+(1-p) H\left(\left[\sum_{i=1}^{l} a_{i} X_{l-i}\right]_{b} \mid X^{l}\right) \\
& =p H\left(\left[\sum_{i=1}^{l} a_{i} X_{l-i}+U\right]_{b} \mid X^{l}\right),
\end{aligned}
$$

where the line follows from the fact that $H\left(\left[\sum_{i=1}^{l} a_{i} X_{l-i}\right]_{b} \mid X^{l}\right)=0$. Therefore, $\limsup _{b \rightarrow \infty} \frac{H\left(\left[X_{l+1}\right]_{b} \mid X^{l}\right)}{b} \geq$ $\lim \sup _{b \rightarrow \infty} \frac{H\left(\left[\sum_{i=1}^{l} a_{i} X_{l-i}+U\right]_{b} \mid X^{l}\right)}{b}$ and $\liminf _{b \rightarrow \infty} \frac{H\left(\left[X_{l+1}\right]_{b} \mid X^{l}\right)}{b} \geq \liminf \inf _{b \rightarrow \infty} \frac{H\left(\left[\sum_{i=1}^{l} a_{i} X_{l-i}+U\right]_{b} \mid X^{l}\right)}{b}$. But,

$$
\begin{aligned}
\liminf _{b \rightarrow \infty} \frac{H\left(\left[\sum_{i=1}^{l} a_{i} X_{l-i}+U\right]_{b} \mid X^{l}\right)}{b} & =\liminf _{b \rightarrow \infty} \int \frac{1}{b} H\left(\left[\sum_{i=1}^{l} a_{i} x_{l-i}+U\right]_{b} \mid X^{l}=x^{l}\right) d \mu\left(x^{l}\right) \\
& \stackrel{(a)}{\geq} \int \liminf _{b \rightarrow \infty} \frac{1}{b} H\left(\left[\sum_{i=1}^{l} a_{i} x_{l-i}+U\right]_{b} \mid X^{l}=x^{l}\right) d \mu\left(x^{l}\right) \\
& \stackrel{(b)}{=} 1,
\end{aligned}
$$

where (a) follows from the Fatou's Lemma, and (b) follows because $U$ has an absolutely continuous distribution. On the other hand,

$$
\liminf _{b \rightarrow \infty} \frac{H\left(\left[\sum_{i=1}^{l} a_{i} X_{l-i}+U\right]_{b} \mid X^{l}\right)}{b} \leq \limsup _{b \rightarrow \infty} \frac{H\left(\left[\sum_{i=1}^{l} a_{i} X_{l-i}+U\right]_{b} \mid X^{l}\right)}{b} \leq 1
$$

where the last inequality follows because $\frac{H\left(\left[\sum_{i=1}^{l} a_{i} X_{l-i}+U\right]_{b} \mid X^{l}\right)}{b} \leq 1$, for all $b$. Therefore, combining this with (27) yields the desired result. That is, $\lim _{b \rightarrow \infty} \frac{H\left(\left[\sum_{i=1}^{l} a_{i} X_{l-i}+U\right]_{b} \mid X^{l}\right)}{b}=1$, and therefore, $\lim _{\sup } \frac{H\left(\left[X_{l+1}\right]_{b} \mid X^{l}\right)}{b} \geq p$ and $\liminf _{b} \frac{H\left(\left[X_{l+1}\right]_{b} \mid X^{l}\right)}{b} \geq p$. These lower bounds combined with the upper bounds derived earlier prove that 
$\bar{d}_{o}(X)=\underline{d}_{o}(X)=p$.

\section{Proof of Theorem 5}

Define process $Z$ as an indicator of the locations where $Y$ is non-zero. That is, $Z_{i}=\mathbb{1}_{Y_{i} \neq 0}$. Then,

$$
\begin{aligned}
& \bar{d}_{k}(X)=\limsup _{b} \frac{H\left(\left[X_{k}\right]_{b} \mid\left[X^{k-1}\right]_{b}\right)}{b} \\
& \leq \limsup _{b} \frac{H\left(\left[X_{k}\right]_{b}, Z_{k-1} \mid\left[X^{k-1}\right]_{b}\right)}{b} .
\end{aligned}
$$

But since process $Y$ is an i.i.d. process and $X^{k-1}$ only depends on $Y_{-l+1}^{k-2}, Z_{k-1}$ is independent of $\left[X^{k-1}\right]_{b}$ and therefore

$$
\begin{aligned}
\frac{H\left(\left[X_{k}\right]_{b}, Z_{k-1} \mid\left[X^{k-1}\right]_{b}\right)}{b}= & \frac{h(p)}{b}+p \frac{H\left(\left[X_{k}\right]_{b} \mid Z_{k-1}=1,\left[X^{k-1}\right]_{b}\right)}{b} \\
& +(1-p) \frac{H\left(\left[X_{k}\right]_{b} \mid Z_{k-1}=0,\left[X^{k-1}\right]_{b}\right)}{b}
\end{aligned}
$$

But,

$$
\underset{b}{\limsup } \frac{H\left(\left[X_{k}\right]_{b} \mid Z_{k-1}=1,\left[X^{k-1}\right]_{b}\right)}{b} \leq \underset{b}{\limsup } \frac{H\left(\left[X_{k}\right]_{b}\right)}{b} \leq 1
$$

Therefore,

$$
\bar{d}_{k}(X) \leq p+(1-p) \limsup _{b} \frac{H\left(\left[X_{k}\right]_{b} \mid Z_{k-1}=0,\left[X^{k-1}\right]_{b}\right)}{b} .
$$

We next prove that $\lim _{k \rightarrow \infty} \lim \sup _{b} \frac{H\left(\left[X_{k}\right]_{b} \mid Z_{k-1}=0,\left[X^{k-1}\right]_{b}\right)}{b}=0$. Note that

$$
H\left(\left[X_{k}\right]_{b} \mid Z_{k-1}=0,\left[X^{k-1}\right]_{b}\right)=H\left(\left[X_{k}\right]_{b} \mid Z_{k-1}=0,\left[X^{k-1}\right]_{b}, Y_{-l+1}^{-1}\right)+I\left(Y_{-l+1}^{-1} ;\left[X_{k}\right]_{b} \mid Z_{k-1}=0,\left[X^{k-1}\right]_{b}\right) .
$$

To prove the desired result, we first show that

$$
\underset{b}{\limsup } \frac{1}{b} H\left(\left[X_{k}\right]_{b} \mid Z_{k-1}=0,\left[X^{k-1}\right]_{b}, Y_{-l+1}^{-1}\right)=0 .
$$

Let $\hat{Y}_{i}, i=0,1, \ldots, k-2$, denote an estimated value of $Y_{i}$, as a function of $\left(\left[X^{k-1}\right]_{b}, Y_{-l+1}^{-1}\right)$, defined as follows. Since $X_{i}=\frac{1}{l} \sum_{j=1}^{l} Y_{i-j}$, we have $Y_{i}=l X_{i+1}-\sum_{j=1}^{l-1} Y_{i-j}$. From this equality, we define

$$
\begin{gathered}
\hat{Y}_{0}=l\left[X_{1}\right]_{b}-\sum_{j=-l+1}^{-1} Y_{i-j}, \\
\hat{Y}_{i}=l\left[X_{i+1}\right]_{b}-\sum_{j=i-l}^{i-2} Y_{j}-\sum_{i=0}^{i-1} \hat{Y}_{j},
\end{gathered}
$$


for $i=1, \ldots, l-1$, and

$$
\hat{Y}_{i}=l\left[X_{i+1}\right]_{b}-\sum_{j=1}^{l-l} \hat{Y}_{i-j}
$$

for $i \geq l$. Define the estimation error process as $E_{i}=Y_{i}-\hat{Y}_{i}$. For $i=0$,

$$
\begin{aligned}
E_{0} & =l\left[X_{1}\right]_{b}-\sum_{j=-l+1}^{-1} Y_{i-j}-\left(l X_{1}-\sum_{j=-l+1}^{-1} Y_{i-j}\right) \\
& =l\left(\left[X_{1}\right]_{b}-X_{1}\right) .
\end{aligned}
$$

Therefore, $\left|E_{0}\right| \leq l 2^{-b}$. For $i=1, \ldots, l-1$,

$$
\left|E_{i}\right| \leq l 2^{-b}+\sum_{i=0}^{i-1}\left|E_{i}\right|
$$

and for $i \geq l$,

$$
\begin{aligned}
\left|E_{i}\right|=\left|Y_{i}-\hat{Y}_{i}\right| & \leq l\left|\left[X_{i+1}\right]_{b}-X_{i+1}\right|+\sum_{j=1}^{l-l}\left|E_{i-j}\right| \\
& \leq l 2^{-b}+\sum_{j=1}^{l-l}\left|E_{i-j}\right| .
\end{aligned}
$$

We prove by induction that $\left|E_{i}\right| \leq 2^{i} l 2^{-b}$, for all $i$. Assume that we know that for $j=0,1, \ldots, i,\left|E_{j}\right| \leq 2^{j} l 2^{-b}$. Let $E_{j}=0$, for $j<0$. Then, for $i+1$,

$$
\left|E_{i+1}\right| \leq l 2^{-b}+\sum_{j=1}^{l}\left|E_{i+1-j}\right| \leq l 2^{-b}+l 2^{-b} \sum_{j=1}^{l} 2^{i+1-j} \leq l 2^{-b} \sum_{j=1}^{i} 2^{j}=l 2^{-b}\left(2^{i+1}-1\right) \leq 2^{i+1} l 2^{-b}
$$

Given the estimates $\left\{\hat{Y}_{i}\right\}_{i}$, and since $X_{k}=\frac{1}{l} \sum_{j=1}^{l} Y_{k-j}$, define an estimate of $X_{k}$ as a function of $\left(\left[X^{k-1}\right]_{b}, Y_{-l+1}^{-1}\right)$ as follows

$$
\hat{X}_{k}=\sum_{j=1}^{l} \hat{Y}_{k-j}
$$

Then, by the triangle inequality,

$$
\left|X_{k}-\hat{X}_{k}\right| \leq \sum_{j=1}^{l}\left|\hat{Y}_{k-j}-Y_{k-j}\right|=\sum_{j=1}^{l}\left|E_{k-j}\right| \leq \frac{1}{l} \sum_{j=1}^{l} 2^{k-j} l 2^{-b} \leq 2^{k} l 2^{-b} .
$$

This proves that given $\left(\left[X^{k-1}\right]_{b}, Y_{-l+1}^{-1}\right)$ there exists an estimate of $X_{k}$, whose distance to $X_{k}$ can be bounded by $2^{k} l 2^{-b}$. Therefore, since $H\left(\left[X_{k}\right]_{b} \mid Z_{k-1}=0,\left[X^{k-1}\right]_{b}, Y_{-l+1}^{-1}\right)=H\left(\left[\hat{X}_{k}+X_{k}-\hat{X}_{k}\right]_{b} \mid Z_{k-1}=0,\left[X^{k-1}\right]_{b}, Y_{-l+1}^{-1}\right)$, the remaining ambiguity in $\left[X_{k}\right]_{b}$ given $\left(\left[X^{k-1}\right]_{b}, Y_{-l+1}^{-1}\right)$ can be bounded by

$$
\log \frac{2^{k} l 2^{-b}}{2^{-b}}=k+\log l
$$

which proves (34). 
We next prove that

$$
\limsup _{b} \frac{1}{b} \frac{I\left(Y_{-l+1}^{-1} ;\left[X_{k}\right]_{b} \mid Z_{k-1}=0,\left[X^{k-1}\right]_{b}\right)}{b} \leq(1-p)^{k-l}
$$

Define for any $k>l$, define random variable $J_{k}$ as an indicator function, which is equal to one if there exists $j$ such that $l+1 \leq j \leq k$, and $Y_{j-l+1}^{j}=0_{k}$. To bound $I\left(Y_{-l+1}^{-1} ;\left[X_{k}\right]_{b} \mid Z_{k-1}=0,\left[X^{k-1}\right]_{b}\right)$, note that

$$
\begin{aligned}
I\left(Y_{-l+1}^{-1} ;\left[X_{k}\right]_{b} \mid Z_{k-1}=0,\left[X^{k-1}\right]_{b}\right) \leq & I\left(Y_{-l+1}^{-1}, J_{k} ;\left[X_{k}\right]_{b} \mid Z_{k-1}=0,\left[X^{k-1}\right]_{b}\right) \\
\leq & 1+I\left(Y_{-l+1}^{-1} ;\left[X_{k}\right]_{b} \mid J_{k}, Z_{k-1}=0,\left[X^{k-1}\right]_{b}\right) \\
\leq & 1+I\left(Y_{-l+1}^{-1} ;\left[X_{k}\right]_{b} \mid J_{k}=0, Z_{k-1}=0,\left[X^{k-1}\right]_{b}\right) \mathrm{P}\left(J_{k}=0\right) \\
& +I\left(Y_{-l+1}^{-1} ;\left[X_{k}\right]_{b} \mid J_{k}=1, Z_{k-1}=0,\left[X^{k-1}\right]_{b}\right) \mathrm{P}\left(J_{k}=1\right) .
\end{aligned}
$$

But conditioned on $J_{k}=1$ and $Z_{k-1}=0, Y_{-l+1}^{-1} \rightarrow\left[X^{k-1}\right]_{b} \rightarrow\left[X_{k}\right]_{b}$, and therefore $I\left(Y_{-l+1}^{-1} ;\left[X_{k}\right]_{b} \mid J_{k}=\right.$ $\left.1, Z_{k-1}=0,\left[X^{k-1}\right]_{b}\right)=0$. On the other hand,

$$
I\left(Y_{-l+1}^{-1} ;\left[X_{k}\right]_{b} \mid J_{k}=0, Z_{k-1}=0,\left[X^{k-1}\right]_{b}\right) \leq b
$$

Moreover, dividing $Y^{k}$ into non-overlapping blocks of size $l$, it follows that if there is no all-zero block of size $l$, then none of these non-overlapping blocks is all-zero. Now since these blocks are non-overlapping and process $Y$ is i.i.d., it shows that

$$
\mathrm{P}\left(J_{k}=0\right) \leq\left((1-p)^{l}\right)^{\left\lfloor\frac{k}{l}\right\rfloor} \leq(1-p)^{k-l}
$$

Therefore,

$$
\limsup _{b} \frac{I\left(Y_{-l+1}^{-1} ;\left[X_{k}\right]_{b} \mid Z_{k-1}=0,\left[X^{k-1}\right]_{b}\right)}{b} \leq(1-p)^{k-l}
$$

Combing this result with (32), (33) and (34) proves that

$$
\bar{d}_{k}(X) \leq p+(1-p)^{k-l+1},
$$

which as $k$ grows to infinity proves that $\bar{d}_{o}(X) \leq p$.

\section{E. Proof of Theorem 7}

We show that for any $\epsilon>0$,

$$
\mathrm{P}\left(\frac{1}{\sqrt{n}}\left\|X_{o}^{n}-\hat{X}_{o}^{n}\right\|_{2}>\epsilon\right) \rightarrow 0
$$

as $n \rightarrow \infty$. Let $X_{o}^{n}=\left[X_{o}^{n}\right]_{b}+q_{o}^{n}$ and $\hat{X}_{o}^{n}=\left[\hat{X}_{o}^{n}\right]_{b}+\hat{q}_{o}^{n}$. By assumption, $A X_{o}^{n}=A \hat{X}_{o}^{n}$, and therefore, $A\left(\left[X_{o}^{n}\right]_{b}-\left[\hat{X}_{o}^{n}\right]_{b}\right)=A\left(q_{o}^{n}-\hat{q}_{o}^{n}\right)$. Note that

$$
\left\|A\left(\left[X_{o}^{n}\right]_{b}-\left[\hat{X}_{o}^{n}\right]_{b}\right)\right\|_{2}=\left\|A\left(q_{o}^{n}-\hat{q}_{o}^{n}\right)\right\|_{2} \leq \sigma_{\max }(A)\left\|q_{o}^{n}-\hat{q}_{o}^{n}\right\|_{2} .
$$


Define the event

$$
\mathcal{E}_{1} \triangleq\left\{\sigma_{\max }(A) \leq \sqrt{n}+2 \sqrt{m}\right\}
$$

From [37], $\mathrm{P}\left(\mathcal{E}_{1}^{c}\right) \leq \mathrm{e}^{-m / 2}$. But,

$$
\left\|q_{o}^{n}-\hat{q}_{o}^{n}\right\|_{2} \leq\left\|q_{o}^{n}\right\|_{2}+\left\|\hat{q}_{o}^{n}\right\|_{2} \leq \sqrt{n} 2^{-b+1}
$$

Hence, conditioned on $\mathcal{E}_{1}$,

$$
\begin{aligned}
\left\|A\left(q_{o}^{n}-\hat{q}_{o}^{n}\right)\right\|_{2} & \leq \sigma_{\max }(A)\left\|q_{o}^{n}-\hat{q}_{o}^{n}\right\|_{2} \\
& \leq n\left(1+2 \sqrt{\frac{m}{n}}\right) 2^{-b+1} .
\end{aligned}
$$

As the next step, we derive a lower bound on $\left\|A\left(\left[X_{o}^{n}\right]_{b}-\left[\hat{X}_{o}^{n}\right]_{b}\right)\right\|_{2}$, which holds with high probability. For a fixed vector $u^{n}$ and for any $\tau \in(0,1)$, by Lemma 4,

$$
\mathrm{P}\left(\left\|A u^{n}\right\|_{2} \leq \sqrt{m(1-\tau)}\left\|u^{n}\right\|_{2}\right) \leq \mathrm{e}^{\frac{m}{2}(\tau+\ln (1-\tau))}
$$

However, $\left[X_{o}^{n}\right]_{b}-\left[\hat{X}_{o}^{n}\right]_{b}$ is not a fixed vector. But as we will show next, with high probability, we can upper bound the number of such vectors possible.

Since $\hat{X}_{o}^{n}$ is the solution of (3), we have

$$
\hat{H}_{k}\left(\left[\hat{X}_{o}^{n}\right]_{b}\right) \leq \hat{H}_{k}\left(\left[X_{o}^{n}\right]_{b}\right)
$$

On the other hand as proved in Appendix A,

$$
\frac{1}{n} \ell_{\mathrm{LZ}}\left(\left[\hat{X}_{o}^{n}\right]_{b}\right) \leq \hat{H}_{k}\left(\left[\hat{X}_{o}^{n}\right]_{b}\right)+\frac{b(k b+b+3)}{\left(1-\epsilon_{n}\right) \log n-b}+\gamma_{n},
$$

where $\gamma_{n}=o(1)$, and does not depend on $\left[\hat{X}_{o}^{n}\right]_{b}$ or $b$, and

$$
\epsilon_{n}=\frac{\log \left(\left(2^{b}-1\right)(\log n) / b+2^{b}-2\right)+2 b}{\log n} .
$$

Combining (40) and (41) and dividing both sides by $b=b_{n}$ yields

$$
\frac{1}{n b_{n}} \ell_{\mathrm{LZ}}\left(\left[\hat{X}_{o}^{n}\right]_{b_{n}}\right) \leq \frac{\hat{H}_{k}\left(\left[X_{o}^{n}\right]_{b_{n}}\right)}{b_{n}}+\frac{k b_{n}+b_{n}+3}{\left(1-\epsilon_{n}\right) \log n-b_{n}}+\frac{\gamma_{n}}{b_{n}}
$$

As the next step, we find an upper bound on $\hat{H}_{k}\left(\left[X_{o}^{n}\right]_{b_{n}}\right) / b_{n}$ that holds with high probability. The upper information dimension of process $X, \bar{d}_{o}(X)$, is defined as $\lim _{k \rightarrow \infty} \bar{d}_{k}(X)$. By Lemma $1, \bar{d}_{k}(X)$ is a non-increasing function of $k$. Therefore, given $\delta_{1}>0$, there exists $k_{\delta_{1}}>0$, such that for any $k>k_{\delta_{1}}$,

$$
\bar{d}_{o}(X) \leq \bar{d}_{k}(X) \leq \bar{d}_{o}(X)+\delta_{1}
$$


By the definition of $d_{k_{\delta_{1}}}(X)$, given $\delta_{2}>0$, there exists $b_{\delta_{2}}$, such that for $b \geq b_{\delta_{2}}$,

$$
\frac{H\left(\left[X_{k_{\delta_{1}}+1}\right]_{b} \mid\left[X^{k_{\delta_{1}}}\right]_{b}\right)}{b} \leq d_{k_{\delta_{1}}}(X)+\delta_{2} .
$$

As a reminder, by the definition, $\hat{H}_{k}\left(x^{n}\right)=H\left(U_{k+1} \mid U^{k}\right)$, where $U^{k+1} \sim p_{k+1}\left(\cdot \mid x^{n}\right)$. Therefore, $\hat{H}_{k}\left(\left[X_{o}^{n}\right]_{b_{n}}\right) / b_{n}$ is a decreasing function of $k$. Therefore, since $k=k_{n}$ by construction is a diverging sequence, for $n$ large enough, $k_{n}>k_{\delta_{1}}$, and

$$
\frac{\hat{H}_{k_{n}}\left(\left[X_{o}^{n}\right]_{b_{n}}\right)}{b_{n}} \leq \frac{\hat{H}_{k_{\delta_{1}}}\left(\left[X_{o}^{n}\right]_{b_{n}}\right)}{b_{n}}
$$

We now prove that, given our choice of parameters, for large values of $n, \hat{H}_{k_{\delta_{1}}}\left(\left[X_{o}^{n}\right]_{b_{n}}\right) / b_{n}=H\left(U_{k_{\delta_{1}}+1} \mid U^{k_{\delta_{1}}}\right) / b_{n}$, where $U^{k_{\delta_{1}}+1} \sim p_{k_{\delta_{1}}+1}\left(\cdot \mid\left[X_{o}^{n}\right]_{b_{n}}\right)$, is close to

$$
\frac{H\left(\left[X_{k_{\delta_{1}}+1}\right]_{b_{n}} \mid\left[X^{k_{\delta_{1}}}\right]_{b_{n}}\right)}{b_{n}}
$$

with high probability.

Since $X$ is $\Psi^{*}$-mixing process, by Theorem 6 , given $\epsilon_{1}>0$, there exists $g \in \mathbb{N}$, only depending on $\epsilon_{1}$ and the distribution of the source process, such that for any $n>6(k+g) / \epsilon_{1}+k$,

$$
\mathrm{P}\left(\left\|p_{k}\left(\cdot \mid\left[X^{n}\right]_{b}\right)-\mu_{k}\right\|_{1} \geq \epsilon_{1}\right) \leq 2^{c \epsilon_{1}^{2} / 8}(k+g) n^{2^{k b}} 2^{\frac{-n c \epsilon_{1}^{2}}{8(k+g)}},
$$

where $c=1 /(2 \ln 2)$. (The empirical distribution function $p_{k_{\delta_{1}+1}}\left(\cdot \mid\left[X^{n}\right]_{b_{n}}\right)$ is defined in Section II-B.) Let

$$
\mathcal{E}_{2} \triangleq\left\{\left\|p_{k_{\delta_{1}+1}}\left(\cdot \mid\left[X^{n}\right]_{b_{n}}\right)-\mu_{k_{\delta_{1}+1}}\right\|_{1} \leq \epsilon_{1} /\left(k_{\delta_{1}}+1\right)\right\}
$$

Letting $k=k_{\delta_{1}}+1$, where $k_{\delta_{1}}>l$, and $b=b_{n}$, for $n$ large enough,

$$
\mathrm{P}\left(\mathcal{E}_{2}^{c}\right) \leq 2^{\frac{c \epsilon_{1}^{2}}{8\left(k_{\delta_{1}}+1\right)}}\left(k_{\delta_{1}}+g+1\right) n^{2^{b_{n}\left(k_{\delta_{1}}+1\right)}} 2^{\frac{-n c \epsilon_{1}^{2}}{8\left(k_{\delta_{1}}+g+1\right)\left(k_{\delta_{1}}+1\right)^{2}}} .
$$

Let $U^{k_{\delta_{1}}+1} \sim p_{k_{\delta_{1}}+1}\left(\cdot \mid\left[x^{n}\right]_{b_{n}}\right)$. Then, conditioned on $\mathcal{E}_{2}$,

$$
\begin{aligned}
\left|\hat{H}_{k_{\delta_{1}}}\left(\left[x^{n}\right]_{b_{n}}\right)-H\left(\left[X_{k_{\delta_{1}}+1}\right]_{b_{n}} \mid\left[X^{k_{\delta_{1}}}\right]_{b_{n}}\right)\right| & =\mid H\left(U_{k_{\delta_{1}}} \mid U^{k_{\delta_{1}}+1}\right)-H\left(\left[X_{k_{\delta_{1}}+1}\right]_{b_{n}} \mid\left[X^{k_{\delta_{1}}}\right]_{b_{n}}\right) \\
& =\left|H\left(U^{k_{\delta_{1}}+1}\right)-H\left(U^{k_{\delta_{1}}}\right)-H\left(\left[X^{k_{\delta_{1}}+1}\right]_{b_{n}}\right)+H\left(\left[X^{k_{\delta_{1}}}\right]_{b_{n}}\right)\right| \\
& \left.\leq \mid H\left(U^{k_{\delta_{1}}+1}\right)-H\left(\left[X^{k_{\delta_{1}}+1}\right]\right]_{b_{n}}\right)|+| H\left(U^{k_{\delta_{1}}}\right)-H\left(\left[X^{k_{\delta_{1}}}\right]_{b_{n}}\right) \mid \\
& \stackrel{(a)}{\leq}-\frac{2 \epsilon_{1}}{k_{\delta_{1}}+1} \log \left(\frac{\epsilon_{1}}{k_{\delta_{1}}+1}\right)+\epsilon_{1} b_{n},
\end{aligned}
$$

where (a) follows from Lemma 5. Dividing both sides of (46) by $b_{n}$ yields

$$
\left|\frac{\hat{H}_{k_{\delta_{1}}}\left(\left[x^{n}\right]_{b_{n}}\right)}{b_{n}}-\frac{H\left(\left[X_{k_{\delta_{1}}+1}\right]_{b_{n}} \mid\left[X^{k_{\delta_{1}}}\right]_{b_{n}}\right)}{b_{n}}\right| \leq-\frac{2 \epsilon_{1}}{\left(k_{\delta_{1}}+1\right) b_{n}} \log \left(\frac{\epsilon_{1}}{k_{\delta_{1}}+1}\right)+\epsilon_{1} .
$$

On the other hand, $b_{n}$ is a diverging sequence of $n$. Therefore, for $n$ large enough, $b_{n} \geq b_{\delta_{2}}$, and as a result, 
combining (43), (44) and (47) yields that, for $n$ large enough, conditioned on $\mathcal{E}_{2}$,

$$
\frac{\hat{H}_{k_{n}}\left(\left[X_{o}^{n}\right]_{b_{n}}\right)}{b_{n}} \leq \bar{d}_{o}(X)+\delta_{3}
$$

where $\delta_{3} \triangleq \delta_{1}+\delta_{2}-\frac{2 \epsilon_{1}}{\left(k_{\delta_{1}}+1\right) b_{n}} \log \frac{\epsilon_{1}}{k_{\delta_{1}}+1}+\epsilon_{1}$ can be made arbitrarily small by choosing $\epsilon_{1}, \delta_{1}$ and $\delta_{2}$ small enough. Furthermore, given our choice of parameters $b_{n}$ and $k_{n}$, from (42) and (48), conditioned on $\mathcal{E}_{2}$,

$$
\frac{1}{n b_{n}} \ell_{\mathrm{LZ}}\left(\left[\hat{X}_{o}^{n}\right]_{b_{n}}\right) \leq \bar{d}_{o}(X)+\delta_{4}
$$

and

$$
\frac{1}{n b_{n}} \ell_{\mathrm{LZ}}\left(\left[X_{o}^{n}\right]_{b_{n}}\right) \leq \bar{d}_{o}(X)+\delta_{4}
$$

where $\delta_{4} \triangleq\left(k_{n} b_{n}+b_{n}+3\right) /\left(\left(1-\epsilon_{n}\right) \log n-b_{n}\right)+\gamma_{n} / b_{n}+\delta_{3}$ can be made arbitrarily small.

Let $\mathcal{C}_{n} \triangleq\left\{\left[x^{n}\right]_{b_{n}}: \ell_{\mathrm{LZ}}\left(\left[x^{n}\right]_{b_{n}}\right) \leq n b_{n}\left(\bar{d}_{o}(X)+\delta_{4}\right)\right\}$. Since the Lempel-Ziv code is a uniquely decodable code, each binary string corresponding to an LZ-coded sequence corresponds to a unique uncoded sequence. Hence, the number of sequences in $\mathcal{C}_{n}$, i.e., the number of quantized sequences satisfying the upper bound of (50), can be bounded as

$$
\begin{aligned}
\left|\mathcal{C}_{n}\right| & \leq \sum_{i=1}^{n b_{n}\left(\bar{d}_{o}(X)+\delta_{4}\right)} 2^{i} \\
& \leq 2^{n b_{n}\left(\bar{d}_{o}(X)+\delta_{4}\right)+1} .
\end{aligned}
$$

Define the event $\mathcal{E}_{3}$ as follows:

$$
\mathcal{E}_{3} \triangleq\left\{\left\|A\left(\left[X_{o}^{n}\right]_{b_{n}}-\left[x^{n}\right]_{b_{n}}\right)\right\|_{2} \geq \sqrt{m(1-\tau)}\left\|\left[X_{o}^{n}\right]_{b_{n}}-\left[x^{n}\right]_{b_{n}}\right\|_{2} ; \forall x^{n} \in[0,1]^{n},\left[x^{n}\right]_{b_{n}} \in \mathcal{C}_{n}\right\}
$$

Assume that we fix $X_{o}^{n}$ and only consider the randomness in drawing the matrix $A$. Then, applying the union bound to (39) and noting the upper bound on the size of $\mathcal{C}_{n}$, derived in (51), we get

$$
\mathrm{P}_{A}\left(\mathcal{E}_{3}^{c}\right) \leq 2^{n b_{n}\left(\bar{d}_{o}(X)+\delta_{4}\right)+2} \mathrm{e}^{\frac{m}{2}(\tau+\ln (1-\tau))} .
$$

Since $X_{o}^{n}$ is a random vector, $\mathrm{P}_{A}\left(\mathcal{E}_{3}^{c}\right)$ is a random variable depending on $X_{o}^{n}$. Taking the expectation of both sides of (52), it follows that

$$
\mathrm{E}_{X_{o}^{n}}\left[\mathrm{P}_{A}\left(\mathcal{E}_{3}^{c}\right)\right] \leq 2^{n b_{n}\left(\bar{d}_{o}(X)+\delta_{4}\right)+2} \mathrm{e}^{\frac{m}{2}(\tau+\ln (1-\tau))}
$$

We next prove with the right choice of parameters, the right hand side of (53) goes to zero. Let

$$
\tau=1-\frac{1}{(\log n)^{2 /(1+v)}},
$$


where $v \in(0,1)$. Then, since by assumption $b=b_{n}=\lceil\log \log n\rceil$, and $m=m_{n} \geq(1+\delta) \bar{d}_{o}(X) n$, it follows that

$$
\begin{aligned}
\mathrm{E}_{X_{o}^{n}}\left[\mathrm{P}_{A}\left(\mathcal{E}_{3}^{c}\right)\right] & \left.\leq 2^{n b_{n}\left(\bar{d}_{o}(X)+\delta_{4}\right)+2} \mathrm{e}^{\frac{m}{2}\left(1-\frac{2}{1+v} \ln \log n\right.}\right) \\
& \leq 2^{n(\log \log n+1)\left(\bar{d}_{o}(X)+\delta_{4}\right)+2} 2^{0.5(1+\delta) \bar{d}_{o}(X) n\left(\log \mathrm{e}-\frac{2}{1+v} \log \log n\right)} \\
& =2^{n(\log \log n)(1+\delta) \bar{d}_{o}(X)\left(\frac{1+\delta_{5}}{1+\delta}-\frac{1}{1+v}+\rho_{n}\right)}
\end{aligned}
$$

where $\delta_{5} \triangleq \delta_{4} / \bar{d}_{o}(X)$ and $\rho_{n}=o(1)$. Note that $\delta_{4}$ and as a result $\delta_{5}$ can be made arbitrarily small for $n$ large enough. Given $\delta>0$, we choose $\delta_{4}$ such that $\delta_{5}<\delta$ and set $v=0.5\left(\delta-\delta_{5}\right) /\left(1+\delta_{5}\right)$. Then, since for this choice of parameters for $n$ large enough,

$$
(1+\delta)\left(\frac{1+\delta_{5}}{1+\delta}-\frac{1}{1+v}+\rho_{n}\right) \leq-\left(\frac{\delta-\delta_{5}}{4}\right),
$$

from (54), we have

$$
\mathrm{E}_{X_{o}^{n}}\left[\mathrm{P}_{A}\left(\mathcal{E}_{3}^{c}\right)\right] \leq 2^{-n(\log \log n) \bar{d}_{o}(X)\left(\delta-\delta_{5}\right) / 4}
$$

On the other hand,

$$
\begin{aligned}
\mathrm{E}_{X_{o}^{n}}\left[\mathrm{P}_{A}\left(\mathcal{E}_{3}^{c}\right)\right] & =\mathrm{E}_{X_{o}^{n}}\left[\mathrm{E}_{A}\left[\mathbb{1}_{\mathcal{E}_{3}^{c}}\right]\right] \\
& =\mathrm{E}_{A}\left[\mathrm{E}_{X_{o}^{n}}\left[\mathbb{1}_{\mathcal{E}_{3}^{c}}\right]\right] \\
& =\mathrm{E}_{A}\left[\mathrm{P}_{X_{o}^{n}}\left(\mathcal{E}_{3}^{c}\right)\right]
\end{aligned}
$$

where the first step follows from Fubini's Theorem. We next prove that $\mathrm{P}_{X_{o}^{n}}\left(\mathcal{E}_{3}^{c}\right)$ converges to zero, almost surely. To prove this result, we employ the Borel Cantelli Lemma. By the Markov inequality, for any $\epsilon>0, \mathrm{P}_{A}\left(\mathrm{P}_{X_{o}^{n}}\left(\mathcal{E}_{3}^{c}\right)>\right.$ $\epsilon) \leq \epsilon^{-1} 2^{-n(\log \log n) \bar{d}_{o}(X)\left(\delta-\delta_{5}\right) / 4}$. Since $\sum_{n=1}^{\infty} \mathrm{P}_{A}\left(\mathrm{P}_{X_{o}^{n}}\left(\mathcal{E}_{3}^{c}\right)>\epsilon\right)<\infty$, by the Borel Cantelli Lemma, as $n$ grows to infinity, $\mathrm{P}_{X_{o}^{n}}\left(\mathcal{E}_{3}^{c}\right)$ converges to zero, almost surely.

By the union bound, $\mathrm{P}\left(\left(\mathcal{E}_{1} \cap \mathcal{E}_{2} \cap \mathcal{E}_{3}\right)^{c}\right) \leq \mathrm{P}\left(\mathcal{E}_{1}^{c}\right)+\mathrm{P}\left(\mathcal{E}_{2}^{c}\right)+\mathrm{P}\left(\mathcal{E}_{3}^{c}\right)$. Clearly $\mathrm{P}\left(\mathcal{E}_{1}^{c}\right) \rightarrow 0$, as $n \rightarrow \infty$. Also, for our choice of parameter $b=b_{n}$, from (45), as $n \rightarrow \infty, \mathrm{P}\left(\mathcal{E}_{2}^{c}\right) \rightarrow 0$ as well. Finally, as we just proved, $\mathrm{P}_{X_{o}^{n}}\left(\mathcal{E}_{3}^{c}\right)$ converges to zero, almost surely. But conditioned on $\mathcal{E}_{1} \cap \mathcal{E}_{2} \cap \mathcal{E}_{3}$, since $\hat{X}_{o}^{n} \in \mathcal{C}_{n}$, from (38),

$$
\sqrt{m(1-\tau)}\left\|X_{o}^{n}-\hat{X}_{o}^{n}\right\|_{2} \leq n\left(1+2 \sqrt{\frac{m}{n}}\right) 2^{-b_{n}+1},
$$

or

$$
\frac{1}{\sqrt{n}}\left\|X_{o}^{n}-\hat{X}_{o}^{n}\right\|_{2} \leq \sqrt{\frac{n}{m(1-\tau)}}\left(1+2 \sqrt{\frac{m}{n}}\right) 2^{-b_{n}+1} .
$$


Since $m / n \leq 1$, we have

$$
\begin{aligned}
\frac{1}{\sqrt{n}}\left\|X_{o}^{n}-\hat{X}_{o}^{n}\right\|_{2} & \leq \frac{3(\log n)^{1 /(1+v)}}{\sqrt{2(1+\delta) \bar{d}_{o}(X)}} 2^{-\log \log n+1} \\
& \leq \frac{3(\log n)^{1 /(1+v)}}{\sqrt{2(1+\delta) \bar{d}_{o}(X)}} 2^{-\log \log n+1} \\
& \leq \frac{6}{\sqrt{2(1+\delta) \bar{d}_{o}(X)}} 2^{-\log \log n(1-1 /(1+v))}
\end{aligned}
$$

which goes to zero as $n$ grows to infinity. This concludes the proof.

\section{F. Proof of Theorem 8}

We need to prove that for any $\epsilon>0$,

$$
\mathrm{P}\left(\frac{1}{\sqrt{n}}\left\|X_{o}^{n}-\hat{X}_{o}^{n}\right\|_{2}>\epsilon\right) \rightarrow 0
$$

as $n \rightarrow \infty$. Throughout the proof $\bar{d}_{o}$ refers to $\bar{d}_{o}(X)$. As before, let $X_{o}^{n}=\left[X_{o}^{n}\right]_{b}+q_{o}^{n}$. As we showed earlier, $\left\|q_{o}^{n}\right\|_{2} \leq \sqrt{n} 2^{-b}$. Since $\hat{X}_{o}^{n}=\arg \min _{u^{n} \in \mathcal{X}_{b}^{n}}\left(\hat{H}_{k}\left(u^{n}\right)+\frac{\lambda}{n^{2}}\left\|A u^{n}-Y_{o}^{m}\right\|_{2}\right)$, we have

$$
\begin{aligned}
\hat{H}_{k}\left(\hat{X}_{o}^{n}\right)+\frac{\lambda}{n^{2}}\left\|A \hat{X}_{o}^{n}-Y_{o}^{m}\right\|_{2}^{2} & \leq \hat{H}_{k}\left(\left[X_{o}^{n}\right]_{b}\right)+\frac{\lambda}{n^{2}}\left\|A q_{o}^{n}\right\|_{2}^{2}, \\
& \leq \hat{H}_{k}\left(\left[X_{o}^{n}\right]_{b}\right)+\frac{\lambda\left(\sigma_{\max }(A)\right)^{2} 2^{-2 b}}{n}
\end{aligned}
$$

Define event $\mathcal{E}_{1}$ as $\mathcal{E}_{1} \triangleq\left\{\sigma_{\max }(A) \leq \sqrt{n}+2 \sqrt{m}\right\}$, where from [37], $\mathrm{P}\left(\mathcal{E}_{1}^{c}\right) \leq \mathrm{e}^{-m / 2}$. Also given $\epsilon>0$, define event $\mathcal{E}_{2}$ as

$$
\mathcal{E}_{2} \triangleq\left\{\frac{1}{b} \hat{H}_{k}\left(\left[X_{o}^{n}\right]_{b}\right) \leq \bar{d}_{o}+\epsilon\right\}
$$

In the proof of Theorem 7, we showed that, for any $\epsilon>0$, given our choice of parameters, $\mathrm{P}\left(\mathcal{E}_{2}^{c}\right)$ converges to zero as $n$ grows to infinity. Conditioned on $\mathcal{E}_{1} \cap \mathcal{E}_{2}$, from (59), we derive

$$
\begin{aligned}
\frac{1}{b} \hat{H}_{k}\left(\hat{X}_{o}^{n}\right)+\frac{\lambda}{b n^{2}}\left\|A \hat{X}_{o}^{n}-Y_{o}^{m}\right\|_{2}^{2} & \leq \bar{d}_{o}+\epsilon+\frac{\lambda 2^{-2 b}}{b}(1+2 \sqrt{m / n})^{2} \\
& \leq \bar{d}_{o}+\epsilon+\frac{9 \lambda 2^{-2 b}}{b}
\end{aligned}
$$

where the last line holds since for $m \leq n, 1+2 \sqrt{m / n} \leq 3$. On the other hand, for $b=b_{n}=\lceil r \log \log n\rceil$ and $\lambda=\lambda_{n}=(\log n)^{2 r}$, we have

$$
\frac{9 \lambda 2^{-2 b}}{b} \leq \frac{9(\log n)^{2 r}}{r(\log n)^{2 r} \log \log n}=\frac{9}{r \log \log n},
$$

which goes to zero as $n$ grows to infinity. For $n$ large enough, $9 /(r \log \log n) \leq \epsilon$, and hence from (60),

$$
\frac{1}{b} \hat{H}_{k}\left(\hat{X}_{o}^{n}\right)+\frac{\lambda}{b n^{2}}\left\|A \hat{X}_{o}^{n}-Y_{o}^{m}\right\|_{2}^{2} \leq \bar{d}_{o}+2 \epsilon
$$


which implies that

$$
\frac{1}{b} \hat{H}_{k}\left(\hat{X}_{o}^{n}\right) \leq \bar{d}_{o}+2 \epsilon
$$

and since $\bar{d}_{o} \leq 1$,

$$
\sqrt{\frac{\lambda}{b n^{2}}}\left\|A \hat{X}_{o}^{n}-Y_{o}^{m}\right\|_{2} \leq \sqrt{1+2 \epsilon}
$$

For $x^{n} \in[0,1]^{n}$, from (A.10) in Appendix A,

$$
\frac{1}{n} \ell_{\mathrm{LZ}}\left(\left[x^{n}\right]_{b}\right) \leq \hat{H}_{k}\left(\left[x^{n}\right]_{b}\right)+\frac{b(k b+b+3)}{\left(1-\epsilon_{n}\right) \log n-b}+\gamma_{n}
$$

where $\epsilon_{n}=o(1)$ and $\gamma_{n}=o(1)$ are both independent of $x^{n}$. Therefore there exists $n_{\epsilon}$, such that for $n>n_{\epsilon}$,

$$
\frac{1}{n b} \ell_{\mathrm{LZ}}\left(\left[x^{n}\right]_{b}\right) \leq \frac{1}{b} \hat{H}_{k}\left(\left[x^{n}\right]_{b}\right)+\epsilon .
$$

On the other hand, conditioned on $\mathcal{E}_{1} \cap \mathcal{E}_{2}, \frac{1}{b} \hat{H}_{k}\left(X_{o}^{n}\right) \leq \bar{d}_{o}+\epsilon$, and $\frac{1}{b} \hat{H}_{k}\left(\hat{X}_{o}^{n}\right) \leq \bar{d}_{o}+2 \epsilon$. Therefore, conditioned on $\mathcal{E}_{1} \cap \mathcal{E}_{2},\left[X_{o}^{n}\right]_{b}, \hat{X}_{o}^{n} \in \mathcal{C}_{n}$, where

$$
\mathcal{C}_{n} \triangleq\left\{\left[x^{n}\right]_{b_{n}}: \frac{1}{n b} \ell_{\mathrm{LZ}}\left(\left[x^{n}\right]_{b_{n}}\right) \leq \bar{d}_{o}+3 \epsilon\right\} .
$$

Define the event $\mathcal{E}_{3}$ as

$$
\mathcal{E}_{3} \triangleq\left\{\left\|A\left(u^{n}-\left[X_{o}^{n}\right]_{b}\right)\right\|_{2} \geq\left\|u^{n}-\left[X_{o}^{n}\right]_{b}\right\|_{2} \sqrt{(1-\tau) m}: \forall u^{n} \in \mathcal{C}_{n}\right\}
$$

where $\tau>0$. As we argued in the proof of Theorem 7, for a fixed input vector $X_{o}^{n}$, by the union bound, we have

$$
\mathrm{P}_{A}\left(\mathcal{E}_{3}^{c}\right) \leq 2^{\left(\bar{d}_{o}+3 \epsilon\right) b n} \mathrm{e}^{\frac{m}{2}(\tau+\ln (1-\tau))} .
$$

Let $\tau=1-(\log n)^{-\frac{2 r}{1+f}}$, where $f>0$. Then, since $b=b_{n} \leq r \log \log n+1$, and $m=m_{n}>2(1+\delta) \bar{d}_{o} n$,

$$
\begin{aligned}
\mathrm{P}_{A}\left(\mathcal{E}_{3}^{c}\right) & \leq 2^{\left(\bar{d}_{o}+3 \epsilon\right)(r \log \log n+1) n} 2^{\frac{m}{2}\left(\log \mathrm{e}-\frac{2 r}{1+f} \log \log n\right)} \\
& =2^{2 r \log \log n\left(n\left(\bar{d}_{o}+3 \epsilon\right)-\frac{m}{2(1+f)}+\alpha_{n}\right)} \\
& \leq 2^{r(\log \log n) n\left(\bar{d}_{o}+3 \epsilon-\left(\frac{1+\delta}{1+f}\right) \bar{d}_{o}+\frac{1}{n} \alpha_{n}\right)}
\end{aligned}
$$

where $\alpha_{n}=o(1)$. For $0<f<\delta$ and $\epsilon<(\delta-f) \bar{d}_{o} / 6(1+f)$, for $n$ large enough, $\bar{d}_{o}+3 \epsilon-\left(\frac{1+\delta}{1+f}\right) \bar{d}_{o}+\frac{1}{n} \alpha_{n}<$ $-(\delta-f) / 2(1+f)$. Let $\nu \triangleq(\delta-f) / 2(1+f)$. Then, for $n$ large enough, $\mathrm{P}_{A}\left(\mathcal{E}_{3}^{c}\right)<2^{-r \nu(\log \log n) n}$. Now applying Fubini's Theorem and the Borel Cantelli Lemma, similar to the proof of Theorem 7, it follows that as $n$ grows to infinity, $\mathrm{P}_{X_{o}^{n}}\left(\mathcal{E}_{3}^{c}\right) \rightarrow 0$, almost surely.

Conditioned on $\mathcal{E}_{1} \cap \mathcal{E}_{2} \cap \mathcal{E}_{3}, \hat{X}_{o}^{n} \in \mathcal{C}_{n}$. Moreover, by the triangle inequality, $\left\|A \hat{X}_{o}^{n}-Y_{o}^{m}\right\|_{2} \geq \| A\left(\hat{X}_{o}^{n}-\right.$ 
$\left.\left[X_{o}^{n}\right]_{b}\right)\left\|_{2}-\right\| A q_{o}^{n} \|_{2}$. Therefore, conditioned on $\mathcal{E}_{1} \cap \mathcal{E}_{2} \cap \mathcal{E}_{3}$, it follows from (62) that

$$
\begin{aligned}
\sqrt{\frac{\lambda(1-\tau) m}{b n^{2}}\left\|\hat{X}_{o}^{n}-\left[X_{o}^{n}\right]_{b}\right\|_{2}} & \leq \sqrt{\frac{\lambda}{b n^{2}}}\left\|A q_{o}^{n}\right\|_{2}+\sqrt{1+2 \epsilon} \\
& \leq \sqrt{\frac{\lambda}{b}}(1+2 \sqrt{m / n}) 2^{-b}+\sqrt{1+2 \epsilon} \\
& \leq \sqrt{\frac{9 \lambda}{b}} 2^{-b}+\sqrt{1+2 \epsilon}
\end{aligned}
$$

or

$$
\frac{1}{\sqrt{n}}\left\|\hat{X}_{o}^{n}-\left[X_{o}^{n}\right]_{b}\right\|_{2} \leq 2^{-b} \sqrt{\frac{9 n}{(1-\tau) m}}+\sqrt{\frac{(1+2 \epsilon) b n}{(1-\tau) \lambda m}} .
$$

Hence, for $\tau=1-(\log n)^{-\frac{2 r}{1+f}}, \lambda=(\log n)^{2 r}$, and $b=\lceil r \log \log n\rceil$,

$$
\frac{1}{\sqrt{n}}\left\|\hat{X}_{o}^{n}-\left[X_{o}^{n}\right]_{b}\right\|_{2} \leq \frac{3+\sqrt{(1+2 \epsilon)(r \log \log n+1)}}{\sqrt{\bar{d}_{o}(1+\delta)}(\log n)^{\frac{r f}{1+f}}},
$$

which can be made arbitrarily small.

\section{G. Proof of Theorem 9}

The proof is very similar to the proof of Theorem 8. Throughout the proof, for ease of notation, $\bar{d}_{o}(X)$ is denoted by $\bar{d}_{o}$. Let $X_{o}^{n}=\left[X_{o}^{n}\right]_{b}+q_{o}^{n}, \epsilon>0, \tau>0$ and $\mathcal{C}_{n} \triangleq\left\{\left[x^{n}\right]_{b}: \frac{1}{n b} \ell_{\mathrm{LZ}}\left(\left[x^{n}\right]_{b}\right) \leq \bar{d}_{o}+3 \epsilon\right\}$. Define events $\mathcal{E}_{1}$, $\mathcal{E}_{2}$ and $\mathcal{E}_{3}$ as done in the proof of Theorem 8, i.e., $\mathcal{E}_{1} \triangleq\left\{\sigma_{\max }(A) \leq \sqrt{n}+2 \sqrt{m}\right\}$, and $\mathcal{E}_{2} \triangleq\left\{\frac{1}{b} \hat{H}_{k}\left(\left[X_{o}^{n}\right]_{b}\right) \leq \bar{d}_{o}+\epsilon\right\}$. and $\mathcal{E}_{3} \triangleq\left\{\left\|A\left(u^{n}-\left[X_{o}^{n}\right]_{b}\right)\right\|_{2} \geq\left\|u^{n}-\left[X_{o}^{n}\right]_{b}\right\|_{2} \sqrt{(1-\tau) m}: \forall u^{n} \in \mathcal{C}_{n}\right\}$. Also, define event $\mathcal{E}_{4}$ as

$$
\mathcal{E}_{4} \triangleq\left\{\left\|Z^{m}\right\|_{2} \leq c_{m}\right\}
$$

Since $\hat{X}_{o}^{n}$ is the minimizer of the cost function in (5), we have

$$
\hat{H}_{k}\left(\hat{X}_{o}^{n}\right)+\frac{\lambda}{n^{2}}\left\|A \hat{X}_{o}^{n}-Y_{o}^{m}\right\|_{2}^{2} \leq \hat{H}_{k}\left(\left[X_{o}^{n}\right]_{b}\right)+\frac{\lambda}{n^{2}}\left\|A q_{o}^{n}+Z^{m}\right\|_{2}^{2}
$$

But $\left\|A q_{o}^{n}+Z^{m}\right\|_{2}^{2} \leq\left\|A q_{o}^{n}\right\|_{2}^{2}+\left\|Z^{m}\right\|_{2}^{2}+2\left\|A q_{o}^{n}\right\|_{2}\left\|Z^{m}\right\|_{2} \leq\left(\sigma_{\max }(A)\right)^{2}\left\|q_{o}^{n}\right\|_{2}^{2}+\left\|Z^{m}\right\|_{2}^{2}+2 \sigma_{\max }(A)\left\|q_{o}^{n}\right\|_{2}\left\|Z^{m}\right\|_{2}$. Since $\left\|q_{o}^{n}\right\|_{2} \leq \sqrt{n} 2^{-b}$ and $m \leq n$, conditioned on $\mathcal{E}_{1} \cap \mathcal{E}_{2} \cap \mathcal{E}_{4}$, we get

$$
\begin{aligned}
\hat{H}_{k}\left(\hat{X}_{o}^{n}\right)+\frac{\lambda}{n^{2}}\left\|A \hat{X}_{o}^{n}-Y_{o}^{m}\right\|_{2}^{2} & \leq \hat{H}_{k}\left(\left[X_{o}^{n}\right]_{b}\right)+\frac{\lambda}{n^{2}}\left((\sqrt{n}+2 \sqrt{m})^{2} n 2^{-2 b}+c_{m}^{2}+2 c_{m}(\sqrt{n}+2 \sqrt{m}) \sqrt{n} 2^{-b}\right) \\
& =\hat{H}_{k}\left(\left[X_{o}^{n}\right]_{b}\right)+\lambda\left((1+2 \sqrt{m / n})^{2} 2^{-2 b}+\left(\frac{c_{m}}{n}\right)^{2}+2 \frac{c_{m}}{n}(1+2 \sqrt{m / n}) 2^{-b}\right) \\
& \leq b\left(\bar{d}_{o}+\epsilon\right)+\lambda\left(9\left(2^{-2 b}\right)+\left(\frac{c_{m}}{n}\right)^{2}+\frac{6 c_{m}}{n} 2^{-b}\right)
\end{aligned}
$$

Dividing both sides of (67), it follows that

$$
\frac{1}{b} \hat{H}_{k}\left(\hat{X}_{o}^{n}\right)+\frac{\lambda}{n^{2} b}\left\|A \hat{X}_{o}^{n}-Y_{o}^{m}\right\|_{2}^{2} \leq \bar{d}_{o}+\epsilon+\frac{\lambda}{b}\left(9\left(2^{-2 b}\right)+\left(\frac{c_{m}}{n}\right)^{2}+\frac{6 c_{m}}{n} 2^{-b}\right) .
$$


By the theorem's assumption, $\lambda=\lambda_{n}=(\log n)^{2 r}$ and $b=b_{n}=\lceil r \log \log n\rceil$. For this choice of the parameters,

$$
\begin{gathered}
\frac{\lambda}{b 2^{2 b}} \leq \frac{(\log n)^{2 r}}{r(\log \log n)(\log n)^{2 r}}=\frac{1}{r \log \log n}, \\
\frac{\lambda c_{m}^{2}}{b n^{2}} \leq \frac{(\log n)^{2 r} c_{m}^{2}}{r(\log \log n) n^{2}}=\frac{1}{r \log \log n}\left(\frac{c_{m}(\log n)^{r}}{n}\right)^{2},
\end{gathered}
$$

and finally

$$
\frac{\lambda c_{m}}{b n 2^{b}} \leq \frac{(\log n)^{2 r} c_{m}}{r(\log \log n) n(\log n)^{r}}=\frac{1}{r \log \log n}\left(\frac{c_{m}(\log n)^{r}}{n}\right) .
$$

Since $c_{m}=O\left(\frac{m}{(\log m)^{r}}\right)$, the right hand sides of (69), (70) and (71) converge to zero as $n$ grows to zero. Therefore, for $n$ large enough, $\frac{\lambda}{b}\left(9\left(2^{-2 b}\right)+\left(\frac{c_{m}}{n}\right)^{2}+\frac{6 c_{m}}{n} 2^{-b}\right)<\epsilon$. The rest of the proof follows exactly as the proof of Theorem 8 .

\section{Conclusions}

In this paper we have studied the problem of universal compressed sensing, i.e., the problem of recovering "structured" signals from their under-determined set of random linear projections without having prior information about the structure of the signal. We have considered structured signals that are modeled by stationary processes. We have generalized Rényi's information dimension and defined information dimension of a stationary process, as a measure of complexity for such processes. We have also calculated the information dimension of some stationary processes, such as Markov processes used to model piecewise constant signals.

We then have introduced the MEP optimization approach for universal compressed sensing. The optimization is based on Occam's Razor and among the signals satisfying the measurements constraints seeks the "simplest" signal. The complexity of a signal is measured in terms of the conditional empirical entropy of its quantized version, which normalized by the quantization level serves as an estimator of the information dimension of the source. We have proved that, asymptotically, for $X_{o}^{n}$ generated by a $\Psi^{*}$-mixing process $X$ with upper information dimension of $\bar{d}_{o}(X)$, MEP requires slightly more that $\bar{d}_{o}(X) n$ random linear measurements to recover the signal. We have also provided an implementable version of MEP, the Lagrangian-MEP algorithm, which is identical to the heuristic algorithm proposed in [21] and [22]. The Lagrangian-MEP algorithm has the same asymptotic performance as the original MEP, and is also robust to the measurement noise. For memoryless sources with a mixture of discretecontinuous distribution, this result shows that both MEP and Lagrangian-MEP achieve the fundamental limits proved in [30], and therefore there is no loss in the performance due to not knowing the source distribution.

\section{APPENDIX A}

\section{CONNECTION BETWEEN $\ell_{\text {LZ }}$ AND $\hat{H}_{k}$}

In this appendix, we adapt the results of [38] to the case where the source is non-binary. Since in this work in most cases we deal with real-valued sources that are quantized at different number of quantization levels and the 
number of of quantization levels usually grows to infinity with blocklength, we need to derive all the constants carefully to make sure that the bounds are still valid, when the size of the alphabet depends on the blocklength.

Consider a finite-alphabet sequence $z^{n} \in \mathcal{Z}^{n}$, where $|\mathcal{Z}|=r$ and $r=2^{b}, b \in \mathbb{N} .^{5}$ Let $N_{\mathrm{LZ}}\left(z^{n}\right)$ denote the number of phrases in $z^{n}$ after parsing it according the Lempel-Ziv algorithm [32].

For $k \in \mathbb{N}$, let

$$
n_{k}=\sum_{j=1}^{k} j r^{j}=\frac{r^{k+1}(k r-(k+1))+r}{(r-1)^{2}} .
$$

Let $N_{\mathrm{LZ}}(n)$ denote the maximum possible number of phrases in a parsed sequence of length $n$. For $n=n_{k}$,

$$
\begin{aligned}
N_{\mathrm{LZ}}\left(n_{k}\right) & \leq \sum_{j=1}^{k} r^{j} \\
& =\frac{r\left(r^{k}-1\right)}{r-1} \\
& =\frac{r^{k+1}(k r-(k+1))}{(r-1)(k r-(k+1))}-\frac{r}{r-1} \\
& \leq \frac{(r-1) n_{k}}{k r-(k+1)} \\
& \leq \frac{n_{k}}{k-1} .
\end{aligned}
$$

Now given $n$, assume that $n_{k} \leq n<n_{k+1}$, for some $k$. It is straightforward to check that for $k \geq 2, n \geq n_{k} \geq r^{k}$. Therefore, if $n>r(1+r)$,

$$
k \leq \frac{\log n}{\log r}
$$

On the other hand, $n<n_{k+1}$, where from (A.1)

$$
\begin{aligned}
n_{k+1} & =\frac{r^{k+2}((k+1) r-(k+2))+r}{(r-1)^{2}} \\
& =\frac{r^{k+2}\left((r-1) \log _{r} n+r-2\right)+r}{(r-1)^{2}} .
\end{aligned}
$$

Therefore,

$$
k+2 \geq \log _{r} \frac{n(r-1)^{2}-r}{(r-1) \log _{r} n+r-2},
$$

or

$$
k \geq\left(1-\epsilon_{n}\right) \frac{\log n}{\log r}
$$

\footnotetext{
${ }^{5}$ Restricting the alphabet size to satisfy this condition is to simplify the arguments, but the results can be generalized to any finite-alphabet source.
} 
where

$$
\epsilon_{n}=\frac{\log \left(\left((r-1) \log _{r} n+r-2\right) r^{2}\right)}{\log n}
$$

To pack the maximum possible number of phrases in a sequence of length $n$, we need to first pack all possible phrases of length smaller than or equal to $k$, then use phrases of length $k+1$ to cover the rest. Therefore,

$$
\begin{aligned}
N_{\mathrm{LZ}}(n) & \leq N_{\mathrm{LZ}}\left(n_{k}\right)+\frac{n-n_{k}}{k+1} \\
& \leq \frac{n_{k}}{k-1}+\frac{n-n_{k}}{k+1} \\
& \leq \frac{n}{k-1} .
\end{aligned}
$$

Combining (A.5) with (A.3), and noting that $\log r=b$, yields

$$
\frac{N_{\mathrm{LZ}}(n)}{n} \leq \frac{b}{\left(1-\epsilon_{n}\right) \log n-b} .
$$

Taking into account the number of bits required for describing the blocklength $n$, the number of phrases $N_{\mathrm{LZ}}$, the pointers and the extra symbols of phrases, we derive

$$
\frac{1}{n} \ell_{\mathrm{LZ}}\left(z^{n}\right)=\frac{1}{n} N_{\mathrm{LZ}} \log N_{\mathrm{LZ}}+\frac{b}{n} N_{\mathrm{LZ}}+\eta_{n}
$$

where

$$
\eta_{n}=\frac{1}{n}\left(\log n+2 \log \log n+\log N_{\mathrm{LZ}}+2 \log \log N_{\mathrm{LZ}}+2\right),
$$

On the other hand, straightforward extension of the analysis presented in [38] to the case of general non-binary alphabets yields

$$
\frac{1}{n} N_{\mathrm{LZ}} \log N_{\mathrm{LZ}} \leq \hat{H}_{k}\left(z^{n}\right)+\frac{N_{\mathrm{LZ}}}{n}((\mu+1) \log (\mu+1)-\mu \log \mu+k \log r),
$$

where $\mu \triangleq N_{\mathrm{LZ}} / n$. But, $(\mu+1) \log (\mu+1)-\mu \log \mu=\log (\mu+1)+\mu \log (1+1 / \mu) \leq \log (\mu+1)+1 / \ln 2<\log \mu+2$. Also, it is easy to show that for any value of $r$ and $z^{n}, n \leq \sum_{i=1}^{N_{\mathrm{LZ}}} l$, or $N_{\mathrm{LZ}}\left(z^{n}\right) \geq \sqrt{2 n}-1$, or $n / N_{\mathrm{LZ}}\left(z^{n}\right) \leq \sqrt{n}$, for $n$ large enough. Therefore, since $\mu^{-1} \log \mu$ is an increasing function of $\mu$,

$$
\frac{\log \mu}{\mu} \leq \frac{\log n}{2 \sqrt{n}}
$$

Hence, combining (A.7), (A.6) and (A.9), we conclude that, for $n$ large enough,

$$
\frac{1}{n} \ell_{\mathrm{LZ}}\left(z^{n}\right) \leq \hat{H}_{k}\left(z^{n}\right)+\frac{b(k b+b+3)}{\left(1-\epsilon_{n}\right) \log n-b}+\gamma_{n},
$$

where $\gamma_{n}=\eta_{n}+\frac{\log n}{2 \sqrt{n}}$, and $\epsilon_{n}$ and $\eta_{n}$ are defined in (A.4) and (A.8), respecctively. Note that $\gamma_{n}=o(1)$ and does not depend on $b$ or $z^{n}$. 


\section{REFERENCES}

[1] D.L. Donoho. Compressed sensing. IEEE Trans. Inf. Theory, 52(4):1289-1306, 2006.

[2] E. J Candès and T. Tao. Near-optimal signal recovery from random projections: Universal encoding strategies? IEEE Trans. Inf. Theory, 52(12):5406-5425, 2006.

[3] E.J. Candès, J. Romberg, and T. Tao. Robust uncertainty principles: exact signal reconstruction from highly incomplete frequency information. IEEE Trans. Inf. Theory, 52(2):489-509, Feb. 2006.

[4] R. G. Baraniuk, V. Cevher, M. F. Duarte, and C. Hegde. Model-based compressive sensing. IEEE Trans. Inf. Theory, 56(4):1982 -2001, Apr. 2010.

[5] V. Chandrasekaran, B. Recht, P. A. Parrilo, and A. S. Willsky. The convex geometry of linear inverse problems. Found. of Comp. Math., 12(6):805-849, 2012.

[6] M. Vetterli, P. Marziliano, and T. Blu. Sampling signals with finite rate of innovation. IEEE Trans. Signal Process., 50(6):1417-1428, Jun. 2002.

[7] B. Recht, M. Fazel, and P. A. Parrilo. Guaranteed minimum rank solutions to linear matrix equations via nuclear norm minimization. SIAM Rev., 52(3):471-501, Apr. 2010.

[8] P. Shah and V. Chandrasekaran. Iterative projections for signal identification on manifolds. In Proc. 49th Annual Allerton Conf. on Commun., Control, and Comp., Sep. 2011.

[9] C. Hegde and R. G. Baraniuk. Signal recovery on incoherent manifolds. IEEE Trans. Inf. Theory, 58(12):7204-7214, 2012.

[10] C. Hegde and R. G. Baraniuk. Sampling and recovery of pulse streams. IEEE Trans. Signal Process., 59(4):1505 -1517, Apr. 2011.

[11] D. L. Donoho, H. Kakavand, and J. Mammen. The simplest solution to an underdetermined system of linear equations. In Proc. IEEE Int. Symp. Inform. Theory (ISIT), pages 1924 -1928, Jul. 2006.

[12] J. Ziv and A. Lempel. A universal algorithm for sequential data compression. IEEE Trans. Inf. Theory, 23(3):337-343, 1977.

[13] D. J. Sakrison. The rate of a class of random processes. IEEE Trans. Inf. Theory, 16:10-16, Jan. 1970.

[14] J. Ziv. Coding of sources with unknown statistics part II: Distortion relative to a fidelity criterion. IEEE Trans. Inf. Theory, 18:389-394, May 1972.

[15] D. L. Neuhoff, R. M. Gray, and L. D. Davisson. Fixed rate universal block source coding with a fidelity criterion. IEEE Trans. Inf. Theory, 21:511-523, May 1972.

[16] D. L. Neuhoff and P. L. Shields. Fixed-rate universal codes for Markov sources. IEEE Trans. Inf. Theory, 24:360-367, May 1978.

[17] D. Donoho. The Kolmogorov sampler. Technical Report 2002-04, Stanford University, Jan. 2002.

[18] T. Weissman, E. Ordentlich, G. Seroussi, S. Verdú, and M. Weinberger. Universal discrete denoising: Known channel. IEEE Trans. Inf. Theory, 51(1):5-28, 2005.

[19] M. Feder, N. Merhav, and M. Gutman. Universal prediction for individual sequences. IEEE Trans. Inf. Theory, 38(4):1258-1270, 1992.

[20] N. Merhav and M. Feder. Universal prediction. IEEE Trans. Inf. Theory, 44(6):2124-2147, 1998.

[21] D. Baron and M. F. Duarte. Universal MAP estimation in compressed sensing. In Proc. 49th Annual Allerton Conf. on Commun., Control, and Comp., Sep. 2011.

[22] D. Baron and M. F. Duarte. Signal recovery in compressed sensing via universal priors. arXiv:1204.2611, 2012.

[23] S. Jalali and A. Maleki. Minimum complexity pursuit. In Proc. 49th Annual Allerton Conf. on Commun., Control, and Comp., pages 1764-1770, Sep. 2011.

[24] S. Jalali, A. Maleki, and R. Baraniuk. Minimum complexity pursuit: Stability analysis. In Proc. IEEE Int. Symp. Inform. Theory, pages 1857-1861, Jul. 2012.

[25] S. Jalali, A. Maleki, and R.G. Baraniuk. Minimum complexity pursuit for universal compressed sensing. IEEE Trans. Inf. Theory, 60(4):2253-2268, April 2014.

[26] S. C. Tornay. Ockham: Studies and Selections. Open Court Publishers, La Salle, IL, Third edition, 1938.

[27] R. J. Solomonoff. A formal theory of inductive inference. Inform. Contr., 7:224-254, 1964.

[28] A. N. Kolmogorov. Logical basis for information theory and probability theory. IEEE Trans. Inf. Theory, 14:662-664, Sep. 1968.

[29] A. Rényi. On the dimension and entropy of probability distributions. Acta Mathematica Academiae Scientiarum Hungarica, 10(1-2):193$215,1959$. 
[30] Y. Wu and S. Verdú. Rényi information dimension: Fundamental limits of almost lossless analog compression. IEEE Trans. Inf. Theory, 56(8):3721 -3748, Aug. 2010.

[31] P. Shields. The Ergodic Theory of Discrete Sample Paths. American Mathematical Society, 1996.

[32] J. Ziv and A. Lempel. Compression of individual sequences via variable-rate coding. IEEE Trans. Inf. Theory, 24(5):530-536, Sep 1978.

[33] R. C. Bradley. Basic properties of strong mixing conditions. a survey and some open questions. Probability surveys, 2(2):107-144, 2005.

[34] S. Geman and D. Geman. Stochastic relaxation, gibbs distributions and the bayesian restoration of images. IEEE Trans. on Pattern Analysis and Machine Intelligence, 6:721-741, Nov 1984.

[35] S. Kirkpatrick, C. D. Gelatt, Jr., and M. P. Vecchi. Optimization by simulated annealing. Science, 220:671-680, 1983.

[36] V. Cerny. Thermodynamical approach to the traveling salesman problem: An efficient simulation algorithm. Journal of Optimization Theory and Applications, 45(1):41-51, Jan 1985.

[37] E. Candès, J. Romberg, and T. Tao. Decoding by linear programming. IEEE Trans. Inf. Theory, 51(12):4203 - 4215, Dec. 2005.

[38] E. Plotnik, M.J. Weinberger, and J. Ziv. Upper bounds on the probability of sequences emitted by finite-state sources and on the redundancy of the Lempel-Ziv algorithm. IEEE Trans. Inf. Theory, 38(1):66-72, Jan 1992. 